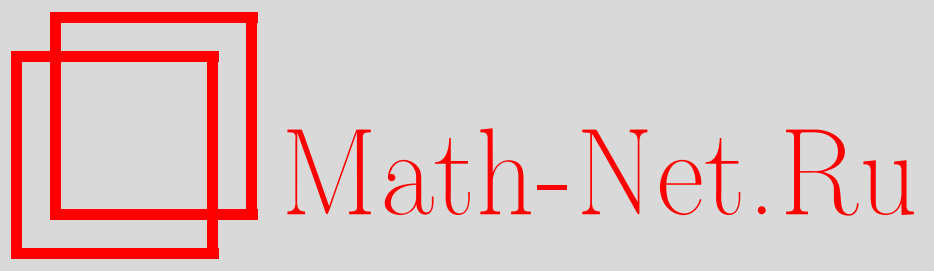

В. Р. Фаталов, Точные асимптотики типа Лапласа для гауссовской меры Боголюбова, ТМФ, 2011, том 168, номep 2, 299-340

DOI: https://doi.org/10.4213/tmf6683

Использование Общероссийского математического портала Math-Net.Ru подразумевает, что вы прочитали и согласны с пользовательским соглашением http://www.mathnet.ru/rus/agreement

Параметры загрузки:

IP : 54.172 .240 .79

26 апреля 2023 г., 16:17:32

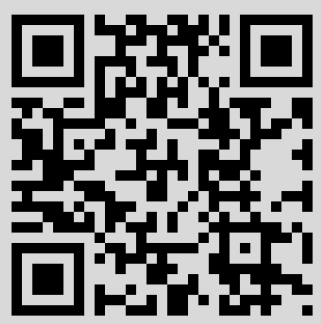




\title{
ТОЧНЫЕ АСИМПТОТИКИ ТИПА ЛАПЛАСА ДЛЯ ГАУССОВСКОЙ МЕРЫ БОГОЛЮБОВА
}

\begin{abstract}
Получены новые асимптотические формулы для двух классов функциональных интегралов типа Лапласа, взятых по мере Боголюбова. В качестве основных функционалов взяты $L^{p}$-функционалы при $0<p<\infty$ и два функционала типа точной верхней грани. В частности, доказаны теоремы об асимптотиках типа Лапласа для моментов $L^{p}$-нормы гауссовского процесса Боголюбова, когда порядок момента становится неограниченно большим. Установлено существование порогового значения $p_{0}=2+4 \pi^{2} / \beta^{2} \omega^{2}$, где $\beta>0$ - обратная температура, $\omega>0$ - собственная частота гармонического осциллятора. Доказано различное поведение исследуемых асимптотик при $0<p<p_{0}$ и $p>p_{0}$. Получены родственные асимптотические результаты о больших уклонениях для меры Боголюбова. Установлено масштабное свойство процесса Боголюбова, позволяющее уменьшить при исследовании число независимых параметров.
\end{abstract}

Ключевые слова: мера Боголюбова, метод Лапласа в банаховом пространстве, принцип больших уклонений, функционал действия.

\section{1. ВВЕДЕНИЕ И ФОРМУЛИРОВКА ОСНОВНЫХ РЕЗУЛЬТАТОВ}

Метод функционального интегрирования, восходящий к работе [1], находит широкое и эффективное применение в таких разделах современной теоретической физики, как квантовая теория поля, статистическая механика и ряд других [2]-[10]. Особый интерес представляет тот важный и распространенный в теории и приложениях случай, когда исследуемый функциональный интеграл берется по некоторой гауссовской мере в пространстве непрерывных функций. Дело в том, что в случае гауссовского функционального интеграла можно провести многие необходимые вычисления, используя хорошо развитые приближенные и асимптотические методы теории гауссовских мер [11]-[17].

Отметим, что выделение и характеризация гауссовской меры, по которой ведется интегрирование в функциональном интеграле, является довольно сложной задачей. В настоящей статье мы рассмотрим один важный и интересный пример подобного рода. Исследуя функциональные интегралы, введенные в работах [18], [19], Санкович доказал [20]-[23], что указанные функциональные интегралы берутся по особой

*Московский государственный университет им. М.В. Ломоносова, Москва, Россия. E-mail: vrfatalov@yandex.ru 
гауссовской мере, которая названа в работе [21] гауссовской мерой Боголюбова. Дадим описание этой меры, придерживаясь работ [20]-[22].

Гауссовский процесс Боголюбова - это стационарный гауссовский процесс $\xi(t)$, $t \in[0, \beta]$, с нулевым средним и ковариационной функцией

$$
B(t-s) \equiv B(t, s):=\mathbf{E} \xi(t) \xi(s)=\frac{1}{2 m \omega \operatorname{sh} \frac{\beta \omega}{2}} \operatorname{ch}\left(\omega|t-s|-\frac{\beta \omega}{2}\right), \quad t, s \in[0, \beta]
$$

Здесь и ниже $\mathbf{E}$ - оператор математического ожидания, взятый относительно вероятности $\mathbf{P}, m>0$ - масса частицы, $\beta>0$ - обратная температура, $\omega>0$ собственная частота гармонического осциллятора [21]. Согласно общему результату из теории гауссовских процессов [24] процесс Боголюбова $\xi(t)$ имеет почти наверное (п. н.) непрерывные траектории на отрезке $[0, \beta]$. Используя формулу (1.1), находим $\mathbf{E}[\xi(\beta)-\xi(0)]^{2}=0$, следовательно, $\xi(0)=\xi(\beta)$ п. н. Учитывая вышеизложенное, заключаем, что траектории гауссовского процесса Боголюбова принадлежат п. н. пространству $C^{0}[0, \beta]$ непрерывных на отрезке $[0, \beta]$ функций $x(t)$ с равномерной метрикой, удовлетворяющих условию $x(0)=x(\beta)$. Распределение $\mu_{\mathrm{B}}$ процесса $\xi(t)$ в пространстве $C^{0}[0, \beta]$ называется гауссовской мерой Боголюбова. Сведения о распределениях случайных процессов в функциональных пространствах можно найти в книге [25]; теория гауссовских мер изложена в работах [26]-[29].

В статье [21] на основе метода работ [11], [12] вычислены приближенно некоторые функциональные интегралы по мере Боголюбова. Приближенные формулы в работе [21] представляют собой квадратурные формулы, которые являются точными для функциональных многочленов. В дополнение к результатам работы [21] в статье [30] были получены приближенные асимптотические формулы для одного класса функциональных интегралов типа Лапласа, взятых по мере Боголюбова. В качестве основного функционала в [30] была взята норма пространства $L^{p}$ при $p>0$.

Проводя дальнейшие исследования асимптотических свойств меры Боголюбова, связанные с одной задачей теории возмущений квантовой теории поля (см. [6], [31]), мы обнаружили, что третьи утверждения в теоремах 1 и 3 из работы [30] справедливы только при $0<p<p_{0}$, где

$$
p_{0} \equiv p_{0}(\beta \omega):=2+\frac{4 \pi^{2}}{\beta^{2} \omega^{2}} .
$$

Оказалось, что вычисление асимптотик интегралов типа Лапласа и вероятностей больших уклонений для меры Боголюбова в случае $L^{p}$-функционалов резко усложняется при $p>p_{0}$.

В настоящей работе мы даем объяснение причины этого явления, связанного с возникновением порогового значения $p_{0}$, а также доказываем утверждения о логарифмических асимптотиках и обсуждаем вид точных асимптотик при $p \geqslant p_{0}$. Кроме того, мы доказываем новые теоремы об асимптотиках типа Лапласа для моментов $L^{p}$-нормы гауссовского процесса Боголюбова, когда порядок момента становится неограниченно большим. Мы также рассматриваем большие уклонения для нормы типа точной верхней грани, т. е. при $p=\infty$. Доказательства полученных в статье результатов проводятся с помощью метода Лапласа в функциональных пространствах для гауссовских мер. 
Связь между мерами Винера и Боголюбова описана в работах [22], [30]. Оказалось, что процесс Боголюбова относится к классу гауссовских процессов винеровского типа в том смысле, что его траектории обладают той же степенью регулярности, что и траектории винеровского процесса: они непрерывны по Гёльдеру порядка $\gamma<1 / 2$ [30] и не удовлетворяют условию Гёльдера порядка $\gamma>1 / 2$ (см. [22], а также [24]). В указанный класс гауссовских процессов типа винеровского входят такие важные в теории и приложениях процессы, как процессы Орнштейна-Уленбека, условные винеровские процессы [6], гауссовские процессы с треугольной ковариационной функцией и ряд других [32], [33].

Перейдем к точной формулировке полученных результатов. Приведенные ниже определения и построения аналогичны изложенным в статье [32], но при этом имеются существенные отличия.

Для фиксированного числа $2<p<\infty$ введем следующие функции, играющие важную роль на протяжении всей статьи:

$$
\begin{gathered}
J(\delta) \equiv J_{p}(\delta):=\int_{\delta}^{1} \frac{d t}{\sqrt{\frac{1-\delta^{2}}{1-\delta^{p}}\left(1-t^{p}\right)-\left(1-t^{2}\right)}}, \\
M(\delta) \equiv M_{p}(\delta):=\int_{\delta}^{1} \frac{t^{p} d t}{\sqrt{\frac{1-\delta^{2}}{1-\delta^{p}}\left(1-t^{p}\right)-\left(1-t^{2}\right)}}, \quad 0<\delta<1 .
\end{gathered}
$$

Из определений получаем неравенства

$$
0<\delta^{p} J(\delta)<M(\delta)<J(\delta), \quad 0<\delta<1 .
$$

Несложно убедиться, что функции $J(\delta)$ и $M(\delta)$ корректно определены и непрерывны, некоторые свойства функции $J(\delta)$ изложены ниже в разделе 3 . Здесь мы отметим, что подынтегральные функции в (1.3) и (1.4) имеют интегрируемые особенности при $t=\delta$ и $t=1$. В лемме 5 настоящей статьи также доказаны следующие соотношения для $p>2$ :

$$
\lim _{\delta \downarrow 0} J_{p}(\delta)=+\infty, \quad \lim _{\delta \uparrow 1} J_{p}(\delta)=\frac{\pi}{\sqrt{p-2}} .
$$

Доопределим по непрерывности

$$
J_{p}(1):=\lim _{\delta \uparrow 1} J_{p}(\delta)=\frac{\pi}{\sqrt{p-2}} .
$$

В разделе 5 доказано следующее важное утверждение.

ЛЕмма 1. Пусть число $p>p_{0}$ биксировано. Тогда уравнение

$$
J_{p}(\delta)=\frac{\beta \omega}{2}, \quad 0<\delta<1,
$$

имеет единственный корень, который мы обозначим через $\delta_{0} \equiv \delta_{0}(p, \beta \omega)$.

Для $p>p_{0}$ положим

$$
b_{0}=b_{0}(p, \beta \omega):=\left(\frac{\beta \omega}{2 M\left(\delta_{0}\right)}\right)^{1 / p}, \quad h_{0}:=b_{0} \delta_{0}, \quad \delta_{\infty}:=\frac{1}{\operatorname{ch} \frac{\beta \omega}{2}} .
$$


Из леммы 12 и формулы (1.5) вытекают неравенства

$$
0<\delta_{\infty}<\delta_{0}<h_{0}<1<b_{0}
$$

Определим также важный параметр

$$
\lambda_{0}=\lambda_{0}(p, \beta \omega):=\beta^{2} \omega^{2} \frac{b_{0}^{2}-h_{0}^{2}}{2\left(b_{0}^{p}-h_{0}^{p}\right)}=\beta^{2} \omega^{2} \frac{1-\delta_{0}^{2}}{2\left(1-\delta_{0}^{p}\right) b_{0}^{p-2}} .
$$

Для $p>p_{0}$ справедливы следующие полезные оценки, которые доказаны ниже в конце этого раздела:

$$
2 \beta \omega \text { th } \frac{\beta \omega}{2} \leqslant p \lambda_{0} \leqslant \omega^{2} \beta^{2}
$$

Важное свойство монотонного убывания по $p \in\left(p_{0}, \infty\right)$ функции $p \lambda_{0}(p, \beta \omega)$ описано в лемме 13.

Положим

$$
\|x\|_{p}:=\left(\int_{0}^{\beta}|x(t)|^{p} d t\right)^{1 / p}, \quad p>0, \quad\|x\|_{\infty}:=\sup _{t \in[0, \beta]}|x(t)| .
$$

Как известно, при $p \geqslant 1$ функционал $\|x\|_{p}$ является нормой в банаховом пространстве $L^{p}[0, \beta]$ функций, интегрируемых в $p$-й степени на отрезке $[0, \beta]$. Точные асимптотики интегралов типа Лапласа для $L^{p}$-нормы, взятых по мере Боголюбова, имеют следующий вид.

Теорема 1. Пусть задано число $0<\alpha<2$. Для $p>0$ положим

$$
q_{0}=q_{0}(p, \alpha)=\frac{2-\alpha}{2}\left(\frac{\alpha}{m \omega^{2}} \beta^{(2-p) / p}\right)^{\alpha /(2-\alpha)}>0 .
$$

Справедливы следующие утверждения.

1. Предположим, что фиксировано число $0<p<2$. Тогда при $u \rightarrow \infty$ выполнено асимптотическое соотношение

$$
\begin{aligned}
\mathbf{E} e^{u\|\xi\|_{p}^{\alpha}} & \equiv \int_{C^{0}[0, \beta]} \exp \left\{u\left(\int_{0}^{\beta}|x(t)|^{p} d t\right)^{\alpha / p}\right\} d \mu_{\mathrm{B}}(x)= \\
& =d_{0} \exp \left\{q_{0} u^{2 /(2-\alpha)}\right\}(1+o(1)),
\end{aligned}
$$

где

$$
d_{0}=d_{0}(p, \alpha)=\frac{2 \sqrt{2-p} \operatorname{sh} \frac{\beta \omega}{2}}{\sqrt{2-\alpha} \operatorname{sh}\left(\frac{\beta \omega}{2} \sqrt{2-p}\right)}>0 .
$$

2. При $и \rightarrow \infty$ имеет место асимптотическое соотношение

$$
\begin{aligned}
& \int_{C^{0}[0, \beta]} \exp \left\{u\left(\int_{0}^{\beta} x^{2}(t) d t\right)^{\alpha / 2}\right\} d \mu_{\mathrm{B}}(x)= \\
& \quad=\exp \left\{\frac{2-\alpha}{2}\left(\frac{\alpha}{m \omega^{2}}\right)^{\alpha /(2-\alpha)} u^{2 /(2-\alpha)}\right\} \frac{4 \operatorname{sh} \frac{\beta \omega}{2}}{\beta \omega \sqrt{2-\alpha}}(1+o(1)) .
\end{aligned}
$$


3. Пусть фиксировано число $2<p<p_{0}$, тогда при $u \rightarrow \infty$ выполнено асимптотическое соотношение

$$
\begin{aligned}
\mathbf{E} e^{u\|\xi\|_{p}^{\alpha}} & \equiv \int_{C^{0}[0, \beta]} \exp \left\{u\left(\int_{0}^{\beta}|x(t)|^{p} d t\right)^{\alpha / p}\right\} d \mu_{\mathrm{B}}(x)= \\
& =d_{1} \exp \left\{q_{0} u^{2 /(2-\alpha)}\right\}(1+o(1)),
\end{aligned}
$$

¿əe

$$
d_{1}=d_{1}(p, \alpha)=\frac{2 \sqrt{p-2} \operatorname{sh} \frac{\beta \omega}{2}}{\sqrt{2-\alpha} \sin \left(\frac{\beta \omega}{2} \sqrt{p-2}\right)}>0 .
$$

4. Пусть фиксировано число $p>p_{0}$, тогда при $u \rightarrow \infty$ выполнено асимптотическое соотношение

$$
\ln \mathbf{E} e^{u\|\xi\|_{p}^{\alpha}}=\ln \int_{C^{0}[0, \beta]} e^{u\|x\|_{p}^{\alpha}} d \mu_{\mathrm{B}}(x)=q_{1} u^{2 /(2-\alpha)}(1+o(1)),
$$

2วe

$$
q_{1}=q_{1}(p, \alpha)=\frac{2-\alpha}{2}\left(\frac{\alpha \beta^{(2+p) / p}}{\lambda_{0} p m}\right)^{\alpha /(2-\alpha)}>0 .
$$

Формулы (1.13)-(1.17) повторяют формулы (1.2)-(1.5) из теоремы 1 работы [30] при $0<p<p_{0}$. Формула (1.18) дает новую константу для логарифмической асимптотики по сравнению с п. 3 упомянутой теоремы при $p>p_{0}$.

Ограничение сверху $\alpha<2$ в теореме 1 есть следствие известной теоремы Ландау-Маркуса-Шеппа-Ферника об экспоненциальной интегрируемости квадрата нормы гауссовского случайного элемента (см. [26]-[29], а также замечание 1 в [30]).

Оценки вероятностей больших уклонений для меры Боголюбова в $L^{p}$-норме приведены в следующей теореме.

ТЕОрема 2. Имеют место следующие утверждения.

1. Пусть фиксировано число $0<p<2$. Тогда при $u \rightarrow \infty$ выполнено асимптотическое соотношение

$$
\begin{aligned}
\mathbf{P}\left\{\|\xi\|_{p}>u\right\} & \equiv \mathbf{P}\left\{\int_{0}^{\beta}|\xi(t)|^{p} d t>u^{p}\right\} \equiv \mu_{\mathrm{B}}\left\{x: \int_{0}^{\beta}|x(t)|^{p} d t>u^{p}\right\}= \\
& =\exp \left\{-\frac{m \omega^{2}}{2} \beta^{(p-2) / p} u^{2}\right\} \frac{d_{2}}{u}(1+o(1))
\end{aligned}
$$

2วe

$$
d_{2}=d_{2}(p)=\frac{\sqrt{2(2-p)} \beta^{(2-p) / 2 p} \operatorname{sh} \frac{\beta \omega}{2}}{\omega \sqrt{\pi m} \operatorname{sh}\left(\frac{\beta \omega}{2} \sqrt{2-p}\right)}>0 .
$$

2. При $и \rightarrow \infty$ справедливо асимптотическое соотношение

$$
\begin{aligned}
\mathbf{P}\left\{\|\xi\|_{2}>u\right\} & \equiv \mathbf{P}\left\{\int_{0}^{\beta} \xi^{2}(t) d t>u^{2}\right\} \equiv \mu_{\mathrm{B}}\left\{x: \int_{0}^{\beta} x^{2}(t) d t>u^{2}\right\}= \\
& =\exp \left\{-\frac{m \omega^{2}}{2} u^{2}\right\} \frac{2 \sqrt{2} \operatorname{sh} \frac{\beta \omega}{2}}{u \beta \omega^{2} \sqrt{\pi m}}(1+o(1)) .
\end{aligned}
$$


3. Пусть фиксировано число $2<p<p_{0}$, тогда при $u \rightarrow \infty$ выполнено асимптотическое соотношение

$$
\begin{aligned}
\mathbf{P}\left\{\int_{0}^{\beta}|\xi(t)|^{p} d t>u^{p}\right\} & \equiv \mu_{\mathrm{B}}\left\{x: \int_{0}^{\beta}|x(t)|^{p} d t>u^{p}\right\}= \\
& =\exp \left\{-\frac{m \omega^{2}}{2} \beta^{(p-2) / p} u^{2}\right\} \frac{d_{3}}{u}(1+o(1))
\end{aligned}
$$

¿əe

$$
d_{3}=d_{3}(p)=\frac{\sqrt{2(p-2)} \beta^{(2-p) / 2 p} \operatorname{sh} \frac{\beta \omega}{2}}{\omega \sqrt{\pi m} \sin \left(\frac{\beta \omega}{2} \sqrt{p-2}\right)}>0 .
$$

4. Пусть фиксировано число $p>p_{0}$, тогда при $u \rightarrow \infty$ выполнено асимптотическое соотношение

$$
\ln \mathbf{P}\left\{\int_{0}^{\beta}|\xi(t)|^{p} d t>u^{p}\right\}=-\frac{m \lambda_{0} p}{2 \beta^{(p+2) / p}} u^{2}(1+o(1)) .
$$

Формулы (1.20)-(1.24) повторяют формулы (1.12)-(1.16) из теоремы 3 работы [30] при $0<p<p_{0}$. Формула (1.25) дает новую константу для логарифмической асимптотики по сравнению с п. 3 указанной теоремы при $p>p_{0}$.

ЗАмЕЧАниЕ 1. Отметим, что точные асимптотики (1.18) для среднего значения и вероятности (1.25) имеют иные степенные множители по $u$ по сравнению с асимптотиками из формул (1.14)-(1.16) и формул (1.21)-(1.23) соответственно. Это связано с тем, что при $p>p_{0}$ соответствующая экстремальная задача для функционала действия в методе Лапласа имеет однопараметрическое многообразие решений (см. ниже лемму 10 и лемму 18). Указанный факт, как и в конечномерном методе Лапласа, оказывает влияние на показатель степенного множителя точной асимптотики (см. теорему 1 в [13], а также работы [34]-[37]). Можно доказать, что при некоторых достаточно простых условиях справедливы следующие асимптотические соотношения при $p>p_{0}$ и $u \rightarrow \infty$ :

$$
\begin{aligned}
& \mathbf{E} e^{u\|\xi\|_{p}^{\alpha}}=\int_{C^{0}[0, \beta]} e^{u\|x\|_{p}^{\alpha}} d \mu_{\mathrm{B}}(x)=c_{1} \exp \left\{q_{1} u^{2 /(2-\alpha)}\right\} u^{1 /(2-\alpha)}(1+o(1)), \\
& \mathbf{P}\left\{\int_{0}^{\beta}|\xi(t)|^{p} d t>u^{p}\right\} \equiv \mu_{\mathrm{B}}\left\{x: \int_{0}^{\beta}|x(t)|^{p} d t>u^{p}\right\}= \\
& =c_{2} \exp \left\{-\frac{m \lambda_{0} p}{2 \beta^{(p+2) / p}} u^{2}\right\}(1+o(1))
\end{aligned}
$$

где $c_{1}=c_{1}(p, m, \beta, \omega)>0$ и $c_{2}=c_{2}(p, m, \beta, \omega)>0$ - некоторые константы.

Строгое доказательство соотношений (1.26) и (1.27) и описание постоянных $c_{1}, c_{2}$ является достаточно сложным и длинным, поэтому его целесообразно изложить в отдельной работе. Здесь мы лишь укажем, что вывод формулы (1.26) основан на теореме 1 из работы [13] при $\operatorname{dim} M=1$ и лемме 18 настоящей статьи, а формула (1.27) доказывается при помощи общей теоремы 1 из работы [37] и леммы 10 настоящей статьи.

Результат теоремы 2 интересно сравнить с точной асимптотикой больших уклонений гауссовского процесса Боголюбова для $L^{\infty}$-функционала, т. е. для точной верхней грани. 
Теорема 3. Пусть фиксировано число $0<\beta_{0}<\beta$. Тогда при $u \rightarrow \infty$ выполнены асимптотические соотношения

$$
\begin{gathered}
\mu_{\mathrm{B}}\left\{x: \sup _{t \in\left[0, \beta_{0}\right]}|x(t)|>u\right\} \equiv \mathbf{P}\left\{\sup _{t \in\left[0, \beta_{0}\right]}|\xi(t)|>u\right\}=2 \mathbf{P}\left\{\sup _{t \in\left[0, \beta_{0}\right]} \xi(t)>u\right\}(1+o(1))= \\
=2 \exp \left\{-m \omega u^{2} \text { th } \frac{\beta \omega}{2}\right\} u \beta_{0} \sqrt{\frac{m}{\pi}}\left(\omega \operatorname{th} \frac{\beta \omega}{2}\right)^{3 / 2}(1+o(1)),
\end{gathered}
$$

$$
\lim _{u \rightarrow \infty} \frac{1}{u^{2}} \ln \mathbf{P}\left\{\sup _{t \in[0, \beta]}|\xi(t)|>u\right\}=\lim _{u \rightarrow \infty} \frac{1}{u^{2}} \ln \mathbf{P}\left\{\sup _{t \in[0, \beta]} \xi(t)>u\right\}=-m \omega \operatorname{th} \frac{\beta \omega}{2} .
$$

Данная теорема доказана ниже в разделе 8. Брать в формуле (1.28) в качестве $\beta_{0}$ число $\beta$, вообще говоря, нельзя. Как видим, показатели степенных множителей по $u$ в правых частях формул из теоремы 2 и соотношения (1.28) разные. Это объясняется тем, что точная асимптотика больших уклонений для функционала типа точной верхней грани (в отличие от интегральных $L^{p}$-функционалов при $0<p<\infty$ ) зависит от гёльдеровских свойств траекторий рассматриваемого гауссовского процесса.

Справедливы следующие аналоги теорем 1 и 2 в случае, когда степень функции $x(t)$ берется без модуля.

Теорема 4. Имеют место следующие утверждения.

1. Пусть фиксированы числа $0<p<p_{0} u 0<\alpha<2$, имеющие следующий вид:

$$
p=\frac{2 k_{1}-1}{2 k_{2}-1}, \quad \alpha=\frac{2 k_{3}-1}{2 k_{4}-1}, \quad k_{j}=1,2, \ldots, \quad j=1,2,3,4 .
$$

Тогда при $и \rightarrow \infty$ справедливо асимптотическое соотношение

$$
\begin{aligned}
& \int_{C^{0}[0, \beta]} \exp \left\{u\left(\int_{0}^{\beta} x^{p}(t) d t\right)^{\alpha / p}\right\} d \mu_{\mathrm{B}}(x)= \\
& =\frac{1}{2} \int_{C^{0}[0, \beta]} \exp \left\{u\left(\int_{0}^{\beta}|x(t)|^{p} d t\right)^{\alpha / p}\right\} d \mu_{\mathrm{B}}(x)(1+o(1)) .
\end{aligned}
$$

2. Пусть биксировано число $0<p<p_{0}$, имеющее вид, заданный в (1.30). Тогда при $u \rightarrow \infty$ справедливо асимптотическое соотношение

$$
\mu_{\mathrm{B}}\left\{\int_{0}^{\beta} x^{p}(t) d t>u^{p}\right\}=\frac{1}{2} \mu_{\mathrm{B}}\left\{\int_{0}^{\beta}|x(t)|^{p} d t>u^{p}\right\}(1+o(1)) .
$$

Далее асимптотика интеграла из правой части формулы (1.31) вычисляется при помощи теоремы 1 , а асимптотика вероятности из правой части формулы (1.32) при помощи теоремы 2. Теорема 4 уточняет теоремы 2 и 4 из статьи [30], в формулировках которых отсутствует условие $p<p_{0}$. Доказательство теоремы 4 аналогично доказательству теорем 2 и 4 из [30].

ЗАмечАниЕ 2. Значения чисел $p>0$ и $0<\alpha<2$ из теоремы 4 подобраны таким образом, что для любого действительного числа $r$ определены однозначно степени $r^{p}, r^{\alpha / p}$, принимающие действительные значения. При этом, в частности, выполнены равенства $(-1)^{p}=-1$ и $\left(\int_{0}^{\beta}(-\xi(t))^{p} d t\right)^{\alpha / p}=-\left(\int_{0}^{\beta} \xi^{p}(t) d t\right)^{\alpha / p}$. 
ЗАМЕЧАНИЕ 3. Для порогового числа $p=p_{0}$ асимптотическое поведение интегралов и вероятностей из теорем 1, 2, 4 нужно исследовать отдельно. Здесь возникает так называемый вырожденный случай (см., в частности, теорему 2.3 в статье [14]). Логарифмические асимптотики при этом имеют тот же вид.

ПреДЛОЖЕНИЕ 1. В случае $p=p_{0}$ справедливъ следующие утверждения.

1. Для $0<\alpha<2$ выполнено асимптотическое равенство

$$
\lim _{u \rightarrow \infty} \frac{1}{u^{2 /(2-\alpha)}} \ln \mathbf{E} \exp \left\{u\left(\int_{0}^{\beta}|\xi(t)|^{p_{0}} d t\right)^{\alpha / p_{0}}\right\}=q_{0}\left(p_{0}, \alpha\right),
$$

где $q_{0}\left(p_{0}, \alpha\right)$ задано в (1.13). Пусть число $p_{0}$ и число $0<\alpha<2$ удовлетворяют равенствам (1.30). Тогда выполнено асимптотическое соотношение

$$
\lim _{u \rightarrow \infty} \frac{1}{u^{2 /(2-\alpha)}} \ln \mathbf{E} \exp \left\{u\left(\int_{0}^{\beta} \xi^{p_{0}}(t) d t\right)^{\alpha / p_{0}}\right\}=q_{0}\left(p_{0}, \alpha\right) .
$$

2. Имеет место равенство

$$
\lim _{u \rightarrow \infty} \frac{1}{u^{2}} \ln \mu_{\mathrm{B}}\left\{\int_{0}^{\beta}|x(t)|^{p_{0}} d t>u^{p_{0}}\right\}=-\frac{m \omega^{2}}{2} \beta^{\left(p_{0}-2\right) / p_{0}} .
$$

Для числа $p_{0}$, удовлетворяющего первому равенству в (1.30), справедливо соотношение

$$
\lim _{u \rightarrow \infty} \frac{1}{u^{2}} \ln \mu_{\mathrm{B}}\left\{\int_{0}^{\beta} x^{p_{0}}(t) d t>u^{p_{0}}\right\}=-\frac{m \omega^{2}}{2} \beta^{\left(p_{0}-2\right) / p_{0}} .
$$

Во многих задачах математической и теоретической физики, теории случайных процессов, математической статистики возникает необходимость приближенного вычисления моментов распределений $L^{p}$-функционалов от гауссовских процессов типа винеровского (см., например, работы [6], [21], [31], [38]). В настоящей статье мы докажем следующий асимптотический результат о моментах распределений $L^{p}$-функционалов для гауссовского процесса Боголюбова. Для $p>0$ положим

$$
q_{2}=q_{2}(p)=\frac{p}{2}-\frac{p}{2} \ln \left(\frac{p}{m \beta \omega^{2}}\right) .
$$

ТЕОРема 5. Справедливы следующие утверждения.

1. Предположим, что фиксировано число $0<p<2$. Тогда при $u \rightarrow \infty$ выполнено асимптотическое соотношение

$$
\int_{C^{0}[0, \beta]}\left[\int_{0}^{\beta}|x(t)|^{p} d t\right]^{u} d \mu_{\mathrm{B}}(x) \equiv \mathbf{E}\left[\int_{0}^{\beta}|\xi(t)|^{p} d t\right]^{u}=d_{5} e^{-q_{2} u} u^{u p / 2} \beta^{u}(1+o(1)),
$$

¿əe

$$
d_{5}=d_{5}(p)=\frac{\sqrt{2(2-p)} \operatorname{sh} \frac{\beta \omega}{2}}{\operatorname{sh}\left(\frac{\beta \omega}{2} \sqrt{2-p}\right)}>0 .
$$

2. При $и \rightarrow \infty$ имеет место равенство

$$
\int_{C^{0}[0, \beta]}\left[\int_{0}^{\beta} x^{2}(t) d t\right]^{u} d \mu_{\mathrm{B}}(x) \equiv \mathbf{E}\left[\int_{0}^{\beta} \xi^{2}(t) d t\right]^{u}=\frac{2 \sqrt{2} \operatorname{sh} \frac{\beta \omega}{2}}{\beta \omega} u^{u}\left(\frac{2}{e m \omega^{2}}\right)^{u}(1+o(1)) .
$$


3. Пусть фиксировано число $2<p<p_{0}$, тогда при $u \rightarrow \infty$ выполнено асимптотическое соотношение

$$
\int_{C^{0}[0, \beta]}\left[\int_{0}^{\beta}|x(t)|^{p} d t\right]^{u} d \mu_{\mathrm{B}}(x) \equiv \mathbf{E}\left[\int_{0}^{\beta}|\xi(t)|^{p} d t\right]^{u}=d_{6} e^{-q_{2} u} u^{u p / 2} \beta^{u}(1+o(1)),
$$

где

$$
d_{6}=d_{6}(p)=\frac{\sqrt{2(p-2)} \operatorname{sh} \frac{\beta \omega}{2}}{\sin \left(\frac{\beta \omega}{2} \sqrt{p-2}\right)}>0
$$

4. Пусть фиксировано число $p>p_{0}$, тогда при $u \rightarrow \infty$ выполнено асимптотическое равенство

$$
\int_{C^{0}[0, \beta]}\left[\int_{0}^{\beta}|x(t)|^{p} d t\right]^{u} d \mu_{\mathrm{B}}(x) \equiv \mathbf{E}\left[\int_{0}^{\beta}|\xi(t)|^{p} d t\right]^{u}=\left(\frac{\beta u}{m}\right)^{u p / 2} \beta^{u} e^{-u q_{3}(p)(1+o(1))}
$$

где

$$
q_{3}=q_{3}(p)=\frac{p}{2}\left(1+\ln \lambda_{0}\right)
$$

а число $\lambda_{0}$ задано в формуле (1.10).

ЗАмЕчАНиЕ 4. Отметим, что в соотношении (1.43) записана логарифмическая асимптотика, так как выражение $1+o(1)$ стоит в показателе последней экспоненты. Можно получить формулу для точной асимптотики интеграла из равенства (1.43) при $p>p_{0}$. Вывод точной асимптотики здесь проводится по схеме доказательства соотношения (1.26).

Теорема 6. Пусть фиксировано число $0<p<p_{0}$, имеющее вид, заданный в (1.30), тогда при $k \rightarrow \infty, k \in \mathbb{N}$, справедливо соотношение

$$
\mathbf{E}\left[\int_{0}^{\beta} \xi^{p}(t) d t\right]^{2 k}=\mathbf{E}\left[\int_{0}^{\beta}|\xi(t)|^{p} d t\right]^{2 k}(1+o(1)) .
$$

Далее асимптотика среднего значения из правой части данной формулы вычисляется при помощи теоремы 5. Отметим интересный факт, состоящий в том, что функция от $k$, записанная в левой части формулы (1.45), не превосходит функции, записанной в правой части этой формулы. Тем не менее эти две функции имеют одинаковую точную асимптотику. Объяснение этого факта дано при доказательстве теоремы 6 в п. 7.8 .

В случае $p=1$ имеем следующее утверждение.

СЛЕДСТВИЕ 1. При $и \rightarrow \infty$ выполнено асимптотическое соотношение

$$
\int_{C^{0}[0, \beta]}\left[\int_{0}^{\beta}|x(t)| d t\right]^{u} d \mu_{\mathrm{B}}(x) \equiv \mathbf{E}\left[\int_{0}^{\beta}|\xi(t)| d t\right]^{u}=\sqrt{2} u^{u / 2}\left(\frac{\beta}{e m \omega^{2}}\right)^{u / 2}(1+o(1)) .
$$


ЗАМЕЧАНИЕ 5. При $p=1$ соотношения (1.45) и (1.46) согласованы друг с другом между собой. В самом деле, используя теорему Фубини и формулу (1.1), легко показать, что случайная величина $\zeta:=\int_{0}^{\beta} \xi(t) d t$ является гауссовской случайной величиной с нулевым средним и дисперсией

$$
\sigma_{0}^{2}(\beta):=\int_{0}^{\beta} \int_{0}^{\beta} \mathbf{E} \xi(t) \xi(s) d t d s=\frac{\beta}{m \omega^{2}} .
$$

Применяя известную формулу для моментов гауссовской случайной величины (см., например, [39]) и выражение для асимптотики гамма-функции (см. п. 6.1.39 в [40]), заключаем, что при $k \rightarrow \infty, k \in \mathbb{N}$, выполнены соотношения

$$
\mathbf{E}\left[\int_{0}^{\beta} \xi(t) d t\right]^{2 k} \equiv \mathbf{E} \zeta^{2 k}=\frac{2^{k}}{\sqrt{\pi}} \Gamma\left(k+\frac{1}{2}\right)\left[\sigma_{0}(\beta)\right]^{2 k}=\sqrt{2}(2 k)^{k} e^{-k}\left[\sigma_{0}(\beta)\right]^{2 k}(1+o(1)) .
$$

Теперь мы можем вычислить двумя разными способами точные асимптотики двух выражений из формулы (1.45) при $p=1$ : при помощи соотношений $(1.47),(1.48)$ и при помощи соотношения (1.46) с $u=2 k$. В результате получаем совпадение указанных двух асимптотик.

Для случая $p=p_{0}$ асимптотическое поведение средних значений из теорем 5,6 нужно исследовать отдельно, так как здесь также возникает вырожденный случай. Логарифмические асимптотики при этом имеют тот же вид.

ПреДЛОжЕНИЕ 2. Имеют место следующие утверждения.

1. При $и \rightarrow \infty$ выполнено асимптотическое соотношение

$$
\int_{C^{0}[0, \beta]}\left[\int_{0}^{\beta}|x(t)|^{p_{0}} d t\right]^{u} d \mu_{\mathrm{B}}(x) \equiv \mathbf{E}\left[\int_{0}^{\beta}|\xi(t)|^{p_{0}} d t\right]^{u}=u^{u p_{0} / 2} \beta^{u} e^{-u q_{2}\left(p_{0}\right)(1+o(1))},
$$

где $q_{2}\left(p_{0}\right)$ задано в (1.37).

2. Для числа $p_{0}$, удовлетворяющего первому равенству в (1.30), справедлива следуюшая асимптотическая формула при $k \rightarrow \infty, k \in \mathbb{N}$ :

$$
\mathbf{E}\left[\int_{0}^{\beta} \xi^{p_{0}}(t) d t\right]^{2 k}=(2 k)^{k p_{0}} \beta^{2 k} e^{-2 k q_{2}\left(p_{0}\right)(1+o(1))} .
$$

Отметим, что в $(1.49),(1.50)$ выражение $1+o(1)$ стоит в показателях экспоненты. Докажем теперь оценки (1.11). Для фиксированных чисел $0<s<r \leqslant \infty$ и функционала (1.12) справедливо неравенство

$$
\|x\|_{s} \leqslant \beta^{1 / s-1 / r}\|x\|_{r}, \quad \text { если }\|x\|_{r}<\infty
$$

(см., например, монографии [41] и [42]); здесь, как обычно, мы полагаем, что $1 / r=0$ при $r=\infty$. Используя утверждение 4 теоремы 2, формулы (1.29), (1.35), (1.51), получаем при $p>p_{0}$ цепочку неравенств

$$
\begin{aligned}
& \lim _{u \rightarrow \infty} \frac{1}{u^{2}} \ln \mathbf{P}\left\{\|\xi\|_{p_{0}}>u \beta^{1 / p_{0}-1 / p}\right\}=-\frac{m \omega^{2}}{2} \beta^{(p-2) / p} \leqslant \lim _{u \rightarrow \infty} \frac{1}{u^{2}} \ln \mathbf{P}\left\{\|\xi\|_{p}>u\right\}= \\
&=-\frac{m \lambda_{0} p}{2 \beta^{(p+2) / p}} \leqslant \lim _{u \rightarrow \infty} \frac{1}{u^{2}} \ln \mathbf{P}\left\{\|\xi\|_{\infty}>\frac{u}{\beta^{1 / p}}\right\}=-\frac{m \omega}{\beta^{2 / p}} \operatorname{th} \frac{\beta \omega}{2} .
\end{aligned}
$$


Отсюда, очевидно, вытекают оценки (1.11). В работе [43] при помощи метода Лапласа доказаны аналоги теорем 5,6 , следствия 1 и предложения 2 для винеровского процесса и броуновского моста.

\section{2. МЕТОД ЛАПЛАСА ДЛЯ ГАУССОВСКИХ МЕР В СЛУЧАЕ ГЛАДКИХ ФУНКЦИОНАЛОВ}

Здесь мы изложим в краткой форме три основных результата, при помощи которых доказываются теоремы 1-6. Более полное изложение имеется в статьях [16], [30].

Пусть $(E,\|\cdot\|)$ - действительное сепарабельное банахово пространство, $\mathcal{E}$ - борелевская $\sigma$-алгебра в $E, E^{*}$ - сопряженное к $E$ пространство, $\langle\cdot, \cdot\rangle-$ линейная форма, устанавливающая двойственность между $E$ и $E^{*}$. Пусть $P_{A}$ - гауссовская мера, определенная на $\mathcal{E}$, имеющая нулевое среднее значение и инъективный ковариационный оператор $A: E^{*} \rightarrow E[29]$.

Обозначим первую и вторую производные Фреше числовой функции $S: E \rightarrow \mathbb{R}$ в точке $x$ через $S^{\prime}(x)$ и $S^{\prime \prime}(x)$. Мы будем предполагать, что производные $S^{\prime}(x)$ и $S^{\prime \prime}(x)$ можно рассматривать как ограниченные линейные операторы из $E$ в $\mathbb{R}$ и из $E$ в $E^{*}$ соответственно, при этом будем употреблять обозначения

$$
S^{\prime}(x)[h]=\left\langle h, S^{\prime}(x)\right\rangle, \quad S^{\prime \prime}(x)[h, v]=\left\langle h, S^{\prime \prime}(x) v\right\rangle, \quad h, v \in E .
$$

Обозначим также через $\operatorname{Dom}(T)$ область определения оператора $T$, через $\operatorname{Ran}(T)-$ область значений оператора $T$, через $I$ - тождественный оператор в $E$. Пусть $\left(H_{A},\|\cdot\|_{A}\right)$ обозначает гильбертово пространство, ассоциированное с ковариационным оператором $A$, т. е. пополнение области значений $\operatorname{Ran}(A)$ оператора $A$ относительно предгильбертовой нормы $\|y\|_{A}:=\sqrt{\langle A y, y\rangle}, y \in E^{*}[29]$.

\section{1. Точные асимптотики для гауссовских интегралов типа Лапласа.} Предположим, что выполнены следующие условия.

D1. Пусть $f$ и $F$ - заданные на $E$ непрерывные действительные функции, такие, что для некоторых постоянных $b_{1}>0, b_{2} \geqslant 0,0 \leqslant b_{3}<1 / 2\|A\|, b_{4} \geqslant 0$ справедливы неравенства

$$
|f(x)| \leqslant b_{1} e^{b_{2}\|x\|^{2}}, \quad F(x) \geqslant-b_{3}\|x\|^{2}-b_{4}, \quad x \in E .
$$

D2. Функционал $\Lambda(x):=F(x)+\left\langle x, A^{-1} x\right\rangle / 2$ достигает своего минимума на множестве $\operatorname{Dom}\left(A^{-1}\right)$ в конечном числе точек $x^{1}, x^{2}, \ldots, x^{m}$. Предположим, что $\inf _{x \in H_{A}}\left[F(x)+\|x\|_{A}^{2} / 2\right]$ также достигается только в точках $x^{1}, x^{2}, \ldots, x^{m}$.

D3. В пространстве $E$ существуют окрестности $U_{i}$ точек $x^{i}$ такие, что функция $F$ трижды дифференцируема по Фреше в точках множеств $U_{i}$, причем $f\left(x^{i}\right) \neq 0$ для всех $i=1,2, \ldots, m$.

D4. Для всех $i=1,2, \ldots, m$ собственные значения $\gamma_{i k}, k=1,2, \ldots$, ядерного оператора $A F^{\prime \prime}\left(x^{i}\right): E \rightarrow E$ таковы, что конечен и не равен нулю детерминант $\operatorname{det} R_{i}:=\prod_{k=1}^{\infty}\left(1+\gamma_{i k}\right)$, где $R_{i}=I+A F^{\prime \prime}\left(x^{i}\right): E \rightarrow E$.

Теорема 7. Пусть $P_{A}$ - гауссовская мера в $E$, имеющая нулевое среднее значение и инбективный ковариационный оператор $A$. 
Пусть выполнены условия D1-D4. Тогда для всех $i=1,2, \ldots, m$ имеем det $R_{i}>$ 0 , выполняются равенства $x^{i}=-A F^{\prime}\left(x^{i}\right)$, и при $u \rightarrow \infty$ справедливо асимптотическое соотношение

$$
\int_{E} f(x) e^{-u^{2} F(x)} d P_{A}(u x)=e^{-u^{2} \Lambda\left(x^{1}\right)} \sum_{i=1}^{m} f\left(x^{i}\right)\left[\operatorname{det} R_{i}\right]^{-1 / 2}(1+o(1)) .
$$

Теорема 7 представляет собой банаховозначный вариант известного результата [14] об асимптотическом разложении гауссовского интеграла типа Лапласа в гильбертовом пространстве (см. также [16]).

\section{2. Точные асимптотики больших уклонений для гауссовских мер.} Рассмотрим более простой случай, когда $E$ представляет собой сепарабельное гильбертово пространство $H$ с нормой $\|\cdot\|$ и скалярным произведением $\langle\cdot, \cdot\rangle$. Предположим, что выполнены следующие условия.

C1. Замкнуто борелевское множество $D=\{x \in H: Q(x) \geqslant 0\}$, где $Q(x)$ - непрерывная действительная функция такая, что или $D$, или множество $H \backslash D$ представимо в виде конечного объединения непересекающихся выпуклых множеств из $H$. Пусть $D$ имеет непустую внутренность и не содержит нулевого элемента пространства $H$.

C2. Квадратичная форма $\left\langle x, A^{-1} x\right\rangle$ достигает своего минимума на $D \cap \operatorname{Dom}\left(A^{-1}\right)$ в конечном числе точек $x^{1}, x^{2}, \ldots, x^{m}$, лежащих на границе множества $D$. Предположим, что $\inf _{x \in D \cap H_{A}}\|x\|_{A}^{2}$ также достигается только в точках $x^{1}, x^{2}, \ldots, x^{m}$.

C3. В гильбертовом пространстве $H$ существуют окрестности $U_{i}$ точек $x^{i}$ такие, что функция $Q$ трижды дифференцируема по Фреше в точках множеств $U_{i}$, причем $\left\langle x^{i}, Q^{\prime}\left(x^{i}\right)\right\rangle \neq 0, i=1,2, \ldots, m$.

C4. Для всех $i=1,2, \ldots, m$ собственные значения $\gamma_{i k}, k=1,2, \ldots$, ядерного оператора $A Q^{\prime \prime}\left(x^{i}\right): H \rightarrow H$ таковы, что не равен нулю $\operatorname{det} R_{i}:=\prod_{k=1}^{\infty}\left(1-\lambda_{i} \gamma_{i k}\right)$, где $R_{i}=I-\lambda_{i} A Q^{\prime \prime}\left(x^{i}\right): H \rightarrow H$ и

$$
\lambda_{i}=\frac{\left\langle x^{1}, A^{-1} x^{1}\right\rangle}{\left\langle x^{i}, Q^{\prime}\left(x^{i}\right)\right\rangle}>0 .
$$

Из этого условия вытекает, что оператор $R_{i}$ является инъективным, $i=1,2, \ldots, m$. Следовательно, согласно альтернативе Фредгольма [41] $\operatorname{Ran}\left(R_{i}\right)=H$ и существует обратный оператор $R_{i}^{-1}$, определенный на всем $H$. В частности, $x^{i} \in \operatorname{Dom}\left(R_{i}^{-1}\right)$, $i=1,2, \ldots, m$.

Теорема 8. Пусть $P_{A}$ - гауссовская мера в гилъбертовом пространстве $H$, имеющая нулевое среднее значение и ингективный ковариационный оператор $A$. Пусть выполнены условия C1-C4. Тогда

$$
x^{i}=\lambda_{i} A Q^{\prime}\left(x^{i}\right), \quad i=1,2, \ldots, m,
$$

и при $и \rightarrow \infty$ справедливо асимптотическое соотношение

$$
P_{A}(u D)=e^{-u^{2}\left\langle x^{1}, A^{-1} x^{1}\right\rangle / 2} \frac{1}{u \sqrt{2 \pi}} \sum_{i=1}^{m}\left|\operatorname{det} R_{i}\left\langle R_{i}^{-1} x^{i}, A^{-1} x^{i}\right\rangle\right|^{-1 / 2}(1+o(1)) .
$$

Теорема 8 доказана в работе [44], банаховозначный вариант этой теоремы изложен в статье [16]. 


\section{3. Логарифмические асимптотики для гауссовских мер и интегралов.} Принцип больших уклонений. Фундаментальный принцип больших уклонений для вероятностных мер и интегралов по ним, берущий свое начало с работы [45], сегодня находит чрезвычайно широкое применение для решения различных асимптотических задач теории вероятностей, статистики, математической и статистической физики, теории чисел, теории информации, математического моделирования и других областей математики, физики, а также естествознания [16], [46]-[53]. Применяя принцип больших уклонений, можно вычислить логарифмические асимптотики интеграла из (2.1) и вероятности из (2.3) в следующих трех случаях, когда вычисление точной асимптотики затруднено:

1) функция $F$ или $Q$ недифференцируемы хотя бы в одной из экстремальных точек $x^{i}$;

2) нарушено условие невырожденности из условий D4 или C4 в одной из точек $x^{i}$, т. е. когда $\operatorname{det} R_{i}=0$,

3) минимум в экстремальной задаче из условий D2 или C2 достигается на конечномерном многообразии ненулевой размерности.

ТЕОРЕма 9 (принцип больших уклонений для гауссовских мер). Имеют место следующие утверждения.

1. Пусть $P_{A}-$ гауссовская мера в банаховом пространстве $E$, имеющая нулевое среднее значение и ингективный ковариационный оператор $A$. Тогда семейство гауссовских мер $\left\{P_{A}(u C), u>0\right\}, C \in \mathcal{E}$, удовлетворяет приниипу больших уклонений с функционалом действия $I_{A}$, имеющим следующий вид:

$$
I_{A}(x)=\left\{\begin{array}{lll}
\|x\|_{A}^{2} / 2, & \text { если } & x \in H_{A}, \\
+\infty, & \text { если } & x \in E \backslash H_{A} .
\end{array}\right.
$$

2. Для любого открытого в $E$ множества $D_{0}$ выполнены неравенства

$$
-\inf _{x \in D_{0}} I_{A}(x) \leqslant \liminf _{u \rightarrow \infty} \frac{1}{u^{2}} \ln P_{A}\left(u D_{0}\right) \leqslant \limsup _{u \rightarrow \infty} \frac{1}{u^{2}} \ln P_{A}(u D) \leqslant-\inf _{x \in D} I_{A}(x),
$$

где $D=\bar{D}_{0}$ - замыкание множества $D_{0}$. В частности, если

$$
\inf _{x \in D_{0}} I_{A}(x)=\inf _{x \in D} I_{A}(x),
$$

mo

$$
\lim _{u \rightarrow \infty} \frac{1}{u^{2}} \ln P_{A}(u D)=-\inf _{x \in D} I_{A}(x) .
$$

3. Для любой непрерывной функции $F: E \rightarrow \mathbb{R}$, удовлетворяющей условию D1, справедливо соотношение

$$
\lim _{u \rightarrow \infty} \frac{1}{u^{2}} \ln \int_{E} e^{-u^{2} F(x)} d P_{A}(u x)=-\inf _{x \in E}\left[F(x)+I_{A}(x)\right] .
$$

ЗАмечАниЕ 6. Соотношение (2.4) верно для многих регулярных множеств, в частности для открытого выпуклого множества $D_{0}$, а также для открытого множества $D_{0}$, замыкание $D$ которого удовлетворяет условию $\mathbf{C 1}$. Укажем также, что

$$
\inf _{x \in D} I_{A}(x)=\frac{1}{2} \inf _{x \in D \cap \operatorname{Ran}(A)}\left\langle x, A^{-1} x\right\rangle
$$


так как $\operatorname{Ran}(A)$ плотно в $\left(H_{A},\|\cdot\|_{A}\right)$, кроме того, если выполнено последнее требование в условии $\mathbf{C 2}$, то минимум в левой и правой частях равенства (2.6) доставляют одни и те же точки.

ЗАмечАниЕ 7. Аналогично, экстремальная задача в правой части равенства (2.5) эквивалентна экстремальной задаче из условия D2 в силу последнего требования в D2, т. е. минимум в обеих задачах одинаков и достигается на одном и том же множестве. Утверждение 3 теоремы 9 часто называют теоремой Варадана, так как вариант этой теоремы впервые появился в статье [45].

На основе соотношения (2.4) проводится вывод логарифмических асимптотик для вероятностей в формулах $(1.25),(1.29),(1.35),(1.36)$. Логарифмические асимптотики для интегралов в формулах (1.18), (1.33), (1.34), (1.43), (1.49), (1.50) доказываются при помощи соотношения (2.5).

\section{3. СВОЙСТВА ФУНКЦИИ $J(\delta)$}

Доказательство теорем 1-6 основано на теоремах 7-9. При этом основную сложность представляет решение экстремальных задач из условий D2 и $\mathbf{C 2}$ для гауссовской меры Боголюбова. Для решения этих экстремальных задач нам понадобятся некоторые свойства функций $J(\delta)$ и $M(\delta)$.

Для фиксированного числа $p>2$ введем следующую функцию:

$$
\phi(t):=\frac{1-t^{2}}{1-t^{p}}, \quad t \in[0,1), \quad \phi(1):=\lim _{t \uparrow 1} \phi(t)=\frac{2}{p} .
$$

ЛЕмма 2. Функция $\phi(t)$ строго монотонно убывает на отрезке $t \in[0,1]$ от значения $\phi(0)=1$ до значения $\phi(1)=2 / p<1$.

ДокАзАТЕЛьство. Производная функции (3.1) имеет вид

$$
\phi^{\prime}(t)=\frac{t}{\left(1-t^{p}\right)^{2}} g(t), \quad g(t):=(2-p) t^{p}+p t^{p-2}-2, \quad t \in[0,1] .
$$

Вычисляя производную, получаем соотношения $g^{\prime}(t)=p(p-2) t^{p-3}\left(1-t^{2}\right)>0$ при $t \in(0,1)$. Таким образом, функция $g(t)$ строго монотонно возрастает на отрезке $[0,1]$ от значения $g(0)=-2$ до значения $g(1)=0$. Следовательно, $g(t)<0$ для всех $t \in[0,1)$. Отсюда и из (3.2) вытекает неравенство $\phi^{\prime}(t)<0$ для всех $t \in(0,1)$. Лемма доказана.

Для фиксированных чисел $0<\delta<t<1$ введем следующую функцию:

$$
\tau(p) \equiv \tau(p, \delta, t):=\frac{1-t^{p}}{1-\delta^{p}}, \quad 0<p<\infty .
$$

Доопределим ее по непрерывности:

$$
\tau(0):=\lim _{p \downarrow 0} \tau(p)=\frac{\ln t}{\ln \delta}, \quad \tau(\infty):=\lim _{p \uparrow \infty} \tau(p)=1 .
$$


ЛЕмма 3. Справедливы следующие утверждения.

1. Функиия

$$
v(x):=\frac{\ln (x+1)}{x}, \quad 0<x<\infty,
$$

строго монотонно убъвает на $(0, \infty)$ от значения $v(0):=\lim _{x \downarrow 0} v(x)=1$ до значения $v(\infty):=\lim _{x \uparrow \infty} v(x)=0$.

2. Функиия $\tau(p)$ строго монотонно возрастает при $0<p<\infty$ от значения $\tau(0)$ до значения $\tau(\infty)=1$.

ДокАЗАТЕЛьСТво. Производная функции (3.4) имеет вид

$$
v^{\prime}(x)=\frac{1}{x^{2}}\left[\frac{x}{x+1}-\ln (x+1)\right], \quad 0<x<\infty .
$$

Учитывая это равенство и известное неравенство $x /(x+1)<\ln (x+1)$ при $x>0$ (см. книгу [40], гл. 4), заключаем, что для всех $x \in(0, \infty)$ справедливо неравенство $v^{\prime}(x)<0$. Это доказывает первое утверждение леммы.

Докажем второе утверждение. Производная функции (3.3) имеет вид

$$
\tau^{\prime}(p)=\frac{1}{\left(1-\delta^{p}\right)^{2}}\left[\left(1-t^{p}\right) \delta^{p} \ln \delta-\left(1-\delta^{p}\right) t^{p} \ln t\right], \quad 0<p<\infty .
$$

Положим

$$
a=\frac{1}{t^{p}}-1, \quad b=\frac{1}{\delta^{p}}-1 .
$$

Напомним, что $0<\delta<t<1$, поэтому $0<a<b<\infty$. Учитывая (3.6) и (3.7), несложно убедиться, что для произвольного фиксированного числа $p \in(0, \infty)$ неравенство $\tau^{\prime}(p)>0$ выполнено тогда и только тогда, когда выполнено неравенство

$$
\frac{\ln (a+1)}{a}>\frac{\ln (b+1)}{b} .
$$

Но последнее неравенство справедливо в силу первого утверждения леммы, так как $0<a<b$. Второе утверждение, а вместе с ним и сама лемма, доказаны.

ЛЕмма 4. При фиксированном $0<\delta<1$ бункция $J_{p}(\delta)$, заданная в $(1.3)$, строго монотонно убавает по $p \in(2, \infty)$. Существует предел

$$
J_{\infty}(\delta):=\lim _{p \uparrow \infty} J_{p}(\delta)=\int_{\delta}^{1} \frac{d t}{\sqrt{t^{2}-\delta^{2}}}=\ln \frac{1+\sqrt{1-\delta^{2}}}{\delta} .
$$

ДокАЗАТЕЛЬСТво. Используя формулы (1.3) и (3.3), получаем следующее равенство для $p \in(2, \infty)$ :

$$
J_{p}(\delta)=\int_{\delta}^{1} \frac{d t}{\sqrt{\left(1-\delta^{2}\right) \tau(p, \delta, t)-\left(1-t^{2}\right)}}, \quad 0<\delta<1 .
$$

В силу второго утверждения леммы 3 для фиксированных $0<\delta<t<1$ подынтегральное выражение в (3.9) является строго монотонно убывающей функцией переменной $p \in(2, \infty)$. Отсюда вытекает первое утверждение леммы. Второе утверждение леммы мы выводим, применяя теорему Лебега о монотонной сходимости. Переходя в равенстве (3.9) к пределу под знаком интеграла при $p \rightarrow \infty$, мы получаем формулу (3.8). Лемма доказана. 
ЛЕмма 5. При любом биксированном $p \in(2, \infty)$ имеют место соотношения

$$
\begin{aligned}
\int_{\delta}^{1} \frac{d t}{\sqrt{(2 / p)\left(1-t^{p}\right)-\left(1-t^{2}\right)}} & \geqslant J_{p}(\delta) \geqslant \int_{\delta}^{1} \frac{d t}{\sqrt{t^{2}-t^{p}}}, \quad 0<\delta<1, \\
\lim _{\delta \downarrow 0} J_{p}(\delta) & =+\infty \\
\lim _{\delta \uparrow 1} J_{p}(\delta) & =\frac{\pi}{\sqrt{p-2}} .
\end{aligned}
$$

ДокАЗАТЕЛЬСТво. Используя формулы (1.3) и (3.1), получаем следующее равенство для $p \in(2, \infty)$ :

$$
J_{p}(\delta)=\int_{\delta}^{1} \frac{d t}{\sqrt{\phi(\delta)\left(1-t^{p}\right)-\left(1-t^{2}\right)}}, \quad 0<\delta<1 .
$$

В силу леммы 2 при $0<\delta<1$ выполнено неравенство $2 / p \leqslant \phi(\delta) \leqslant 1$. Отсюда и из соотношения (3.13) вытекают неравенства (3.10). Переходя в последнем неравенстве из (3.10) к пределу при $\delta \downarrow 0$, получаем соотношение (3.11), поскольку $\int_{0}^{1} d t / \sqrt{t^{2}-t^{p}}=+\infty$. Доказательство формулы (3.12) проведем по той же схеме, что и доказательство леммы 2 в работе [32]. Сделав в интеграле (1.3) замену переменной $s=t^{p}$, получим равенство

$$
J_{p}(\delta)=\frac{1}{p} \int_{\delta^{p}}^{1} \frac{s^{(1-p) / p} d s}{\sqrt{s^{2 / p}-\left(s-\delta^{p}\right) /\left(1-\delta^{p}\right)+(s-1) \delta^{2} /\left(1-\delta^{p}\right)}} .
$$

Сделав в последнем интеграле замену переменной $y=\left(s-\delta^{p}\right) /\left(1-\delta^{p}\right)$ и положив $\varepsilon=1-\delta^{p}$, находим

$$
J_{p}(\delta)=\frac{\varepsilon}{p} K(\varepsilon)
$$

где

$$
K(\varepsilon):=\int_{0}^{1} \frac{(y \varepsilon+1-\varepsilon)^{(1-p) / p} d y}{\sqrt{(y \varepsilon+1-\varepsilon)^{2 / p}-y+(y-1)(1-\varepsilon)^{2 / p}}} .
$$

Используя это определение и оценки $1-\varepsilon \leqslant y \varepsilon+1-\varepsilon \leqslant 1$, справедливые при $0 \leqslant y \leqslant 1$, убеждаемся после простых преобразований, что выполнено соотношение

$$
K(\varepsilon)=K_{1}(\varepsilon)(1+o(1)), \quad \varepsilon \downarrow 0,
$$

где

$$
K_{1}(\varepsilon):=\int_{0}^{1} \frac{d x}{\sqrt{(1-x \varepsilon)^{2 / p}-(1-x)-x(1-\varepsilon)^{2 / p}}} .
$$

Применяя формулу Тейлора, получаем для $x \in[0,1]$ равенство при $\varepsilon \downarrow 0$ :

$$
(1-x \varepsilon)^{2 / p}-(1-x)-x(1-\varepsilon)^{2 / p}=\frac{p-2}{p^{2}} x(1-x) \varepsilon^{2}+O\left(\varepsilon^{3}\right) .
$$

Из формул (3.18), (3.19) и определения бета-функции [40] вытекает, что при $\varepsilon \downarrow 0$

$$
K_{1}(\varepsilon)=\frac{p}{\varepsilon \sqrt{p-2}} \int_{0}^{1} \frac{d x}{\sqrt{x(1-x)}}(1+o(1))=\frac{\pi p}{\varepsilon \sqrt{p-2}}(1+o(1)) .
$$

Используя формулы (3.15), (3.17) и (3.20), получаем соотношение (3.12). Лемма доказана. 


\section{4. МАСШТАБНОЕ СВОЙСТВО ПРОЦЕССА БОГОЛЮБОВА И ВЫБОР ГИЛЬБЕРТОВА ФУНКЦИОНАЛЬНОГО ПРОСТРАНСТВА}

Доказательства первых трех утверждений в теоремах 1 и 2 при $p<p_{0}$ изложены в статье [30] и остаются без изменений. В настоящей статье мы дадим доказательства четвертых пунктов этих теорем, а также доказательства теорем 3-6. При этом некоторые пояснения и формулировки будут приведены для общего случая $p>0$, в частности решения экстремальных задач из условий D2, C2 мы изложим для $p>0$.

Как уже было сказано, случай $p>p_{0}$ оказался наиболее сложным для исследования рассматриваемых асимптотик. Учитывая это, ради упрощения вычислений и записи доказательств мы используем так называемое масштабное свойство процесса Боголюбова, которое интересно и важно само по себе.

Назовем процесс $\xi(t), t \in[0, \beta]$, рассмотренный в разделе 1 (см. (1.1)), процессом Боголюбова с параметрами $(m, \beta, \omega)$. Определим гауссовский процесс

$$
\eta(t):=\frac{\sqrt{m}}{\sqrt{\beta}} \xi(\beta t), \quad t \in[0,1] .
$$

Сформулируем масштабное свойство процесса Боголюбова.

ПРЕДЛОЖЕНИЕ 3. Гауссовский процесс (4.1) представляет собой прощесс Боголюбова с параметрами $\left(1,1, \omega_{1}\right)$, где

$$
\omega_{1}:=\beta \omega>0
$$

ДокАЗАТЕЛьство. Используя формулы (1.1) и (4.1), заключаем, что гауссовский процесс $\eta(t), t \in[0,1]$, имеет нулевое среднее значение и ковариационную функцию

$$
\mathbf{E} \eta(t) \eta(s)=\frac{m}{\beta} \mathbf{E} \xi(\beta t) \xi(\beta s)=\frac{1}{2 \beta \omega \operatorname{sh} \frac{\beta \omega}{2}} \operatorname{ch}\left(\beta \omega|t-s|-\frac{\beta \omega}{2}\right), \quad t, s \in[0,1] .
$$

Искомое утверждение очевидно, вытекает из формул (4.2), (4.3).

Как будет показано ниже, в силу предложения 3 теоремы типа 1-6 достаточно доказать для процесса Боголюбова $\eta(t)$. Изложим сначала доказательство теоремы 2 , основанное на теоремах 8,9 .

ЛЕмма 6. Для любых $p>0, u>0$ выполнено равенство

$$
\mathbf{P}\left\{\int_{0}^{\beta}|\xi(t)|^{p} d t>u^{p}\right\}=\mathbf{P}\left\{\int_{0}^{1}|\eta(s)|^{p} d s>v^{p}\right\}, \quad v=\frac{u \sqrt{m}}{\beta^{(2+p) / 2 p}} .
$$

ДоказАтЕльство. Сделав в первом интеграле в (4.4) замену переменной $t=\beta s$ и используя формулу (4.1), получаем равенство (4.4).

Таким образом, нам достаточно доказать вариант теоремы 2 для процесса Боголюбова (4.1) с параметрами $\left(1,1, \omega_{1}\right)$ и параметром $v \rightarrow \infty$.

Возьмем в качестве гильбертова пространства $H$ обычное гильбертово пространство $L^{2} \equiv L^{2}[0,1]$ интегрируемых в квадрате на $[0,1]$ действительных измеримых 
функций со скалярным произведением и нормой, заданными следующим образом: $\langle x, y\rangle=\int_{0}^{1} x(t) y(t) d t$ и $\|x\|=\left(\int_{0}^{1} x^{2}(t) d t\right)^{1 / 2}$ для $x, y \in L^{2}[0,1]$.

Обозначим через $\mu_{\eta}$ распределение процесса $\eta(t)$ в пространстве $C^{0}[0,1]$. Мы будем рассматривать гауссовскую меру $\mu_{\eta}$, заданную в пространстве $L^{2}[0,1]$, имея в виду, что эта мера сосредоточена на $C^{0}[0,1]$. Как известно, в этом случае мера $\mu_{\eta}$ имеет нулевое среднее значение и ковариационный оператор $A_{\eta}: L^{2} \rightarrow L^{2}$ интегрального типа (см., например, [28], [30]): для $x \in L^{2}$

$$
A_{\eta} x(t)=\int_{0}^{1}[\mathbf{E} \eta(t) \eta(s)] x(s) d s=\frac{1}{2 \omega_{1} \operatorname{sh} \frac{\omega_{1}}{2}} \int_{0}^{1} \operatorname{ch}\left(\omega_{1}|t-s|-\frac{\omega_{1}}{2}\right) x(s) d s .
$$

Пусть $C^{2}[0,1]$ - множество дважды дифференцируемых на $[0,1]$ функций $x(t)$.

Лемма 7. Область значений ковариационного оператора $A_{\eta}$, определенного в формуле (4.5), имеет вид

$$
\operatorname{Ran}\left(A_{\eta}\right)=\left\{x \in C^{2}[0,1]: x(0)=x(1), x^{\prime}(0)=x^{\prime}(1)\right\} .
$$

Обратный оператор задается формулой

$$
\left[A_{\eta}^{-1} y\right](t)=-y^{\prime \prime}(t)+\omega_{1}^{2} y(t), \quad y \in \operatorname{Ran}\left(A_{\eta}\right), \quad t \in[0,1]
$$

ДокАЗАТЕЛьство. Утверждения леммы получаются в результате двукратного дифференцирования по $t$ равенства $y(t)=A_{\eta} x(t)$.

Лемма 8. Собственные числа $\left\{\nu_{n}\right\}_{n=-\infty}^{\infty}$ ковариационного оператора $A_{\eta}$ имеют следующий вид:

$$
\nu_{n}=\frac{1}{\omega_{1}^{2}+(2 \pi n)^{2}}, \quad n=0, \pm 1, \pm 2, \ldots,
$$

им соответствуют ортонормированные в пространстве $L^{2}[0,1]$ собственные функuиu

$$
\varphi_{0}(t)=1 ; \quad \varphi_{n}(t)=\sqrt{2}\left\{\begin{array}{lll}
\cos (2 \pi n t), & \text { если } & n>0 \\
\sin (2 \pi n t), & \text { если } & n<0 .
\end{array}\right.
$$

ДоказАтельство. Рассмотрим задачу на собственные числа для ядерного опеpaтора $A_{\eta}$ :

$$
A_{\eta} \varphi=\nu \varphi, \quad \varphi \in L^{2} .
$$

Поскольку оператор $A_{\eta}$ является инъективным и положительным, в уравнении (4.10) параметр $\nu>0$. Согласно соотношениям (4.6), (4.7) уравнение (4.10) равносильно следующей граничной задаче на собственные числа:

$$
\begin{gathered}
\varphi^{\prime \prime}(t)+\left(\frac{1}{\nu}-\omega_{1}^{2}\right) \varphi(t)=0, \quad t \in[0,1], \\
\varphi(0)=\varphi(1), \quad \varphi^{\prime}(0)=\varphi^{\prime}(1) .
\end{gathered}
$$

Используя № 2.9 из справочника [54], заключаем, что решениями данной задачи являются собственные числа (4.8) и собственные функции (4.9). Лемма доказана. 
ЗАМЕЧАНИЕ 8. Согласно известной осцилляционной теореме для широкого круга задач на собственные числа с периодическими краевыми условиями [55] основная собственная функция $\varphi_{0}(t)$ не имеет нулей на отрезке $[0,1]$, а число нулей двух собственных функций $\varphi_{n}(t), n= \pm k, k>0$, на полуоткрытом интервале $(0,1]$ равно $2 k$. В случае задачи (4.11) этот факт можно установить и непосредственно, учитывая формулу (4.9). Иначе говоря, для широкого круга задач на собственные числа для дифференциального уравнения второго порядка с периодическими краевыми условиями справедлив следующий вывод: менъшему из двух различных собственных чисел соответствует собственная функиия, имеющая меньшее число нулей.

\section{5. ДОКАЗАТЕЛЬСТВО П. 3 И П. 4 ТЕОРЕМЫ 2}

Мы докажем третье и четвертое утверждения теоремы 2 , применяя теоремы 8 и 9 , где мы будем полагать $E=H=L^{2}[0,1], P_{A}=\mu_{\eta}, A=A_{\eta}$. Некоторые утверждения, приведенные ниже, мы формулируем в достаточно общем виде, рассматривая случай $p>0$ и имея целью обоснование соотношения (1.27) из замечания 1.

5.1. Выбор основного функционала. Учитывая лемму 6, возьмем в качестве функционала $Q$, участвующего в формулировке теоремы 8 , следующий функционал:

$$
G(x):=\int_{0}^{1}|x(t)|^{p} d t-1, \quad x \in L^{2}, \quad p>0 .
$$

Обозначим через $\mathcal{X}$ множество функций из $C^{0}[0,1]$, имеющих конечное число нулей. Очевидно, множество $\mathcal{X}$ всюду плотно в $C^{0}[0,1]$, а следовательно, и в $L^{2}$. Отметим, что $0 \notin \mathcal{X}$ (здесь функция, тождественно равная нулю на $[0,1]$, также обозначена через 0$)$.

Ниже мы используем определение производной Фреше, данное в работе [56], где допускается, чтобы рассматриваемый оператор был определен всего лишь на всюду плотном подмножестве банахова пространства; значения, равные $\pm \infty$, возможны (см. определение 1 в [57]).

ЛЕмма 9. Имеют место следующие утверждения.

1. При р > 0 функиионал $G$ трижды дифферениируем по Фреше в каждой точке $x \in \mathcal{X}, u$ его производные равны

$$
\begin{aligned}
G^{\prime}(x) & =p|x(t)|^{p-1} \operatorname{sign} x(t), \\
G^{\prime \prime}(x) & =p(p-1)|x(t)|^{p-2} \\
G^{\prime \prime \prime}(x)[h, g, v] & =\int_{0}^{1} p(p-1)(p-2)|x(t)|^{p-3} \operatorname{sign} x(t) h(t) g(t) v(t) d t, \quad h, g, v \in L^{2} .
\end{aligned}
$$

2. Функционал $G$ является выпуклым функиионалом при $p \geqslant 1$.

3. При $p \geqslant 2$ производные (5.2), (5.3) задают ограниченные линейные операторы при $x \in C^{0}[0,1]$.

ДокАЗАТЕЛЬство первых двух утверждений леммы простое, его можно найти в [30]. Третье утверждение непосредственно вытекает из вида формул (5.2), (5.3). 
5.2. Решение экстремальной задачи. Положим

$$
D_{0}=\left\{x \in L^{2}: G(x)>0\right\}, \quad D=\left\{x \in L^{2}: G(x) \geqslant 0\right\} .
$$

В силу утверждения 2 леммы 9 множество $L^{2} \backslash D$ является выпуклым при $p \geqslant 1$. Очевидно, что множество $D$ имеет непустую внутренность и не содержит ноль. Таким образом, множество $D$ удовлетворяет условию $\mathbf{C 1}$ при $p \geqslant 1$, и к нему применимо замечание 6.

Решим экстремальную задачу из условия $\mathbf{C 2}$ для множества $D$, определенного в (5.4), и ковариационного оператора $A_{\eta}$, заданного в (4.5). Определим постоянную функцию

$$
e_{0}(t) \equiv 1, \quad t \in[0,1]
$$

Для фиксированного $p>p_{0}$ введем функцию от $s, h_{0} \leqslant s \leqslant b_{0}$,

$$
\theta(s) \equiv \theta\left(s, p, \omega_{1}\right):=\frac{1}{\omega_{1}} \int_{\delta_{0}}^{s / b_{0}} \frac{d z}{\sqrt{\left(1-\delta_{0}^{2}\right)\left(1-\delta_{0}^{p}\right)^{-1}\left(1-z^{p}\right)-\left(1-z^{2}\right)}},
$$

где положительные постоянные $h_{0}, b_{0}, \delta_{0}, \lambda_{0}$ заданы посредством формул (1.7), $(1.8),(1.10), \omega_{1}$ определено в (4.2). Легко убедиться, что функция $\theta(s)$ монотонно возрастает при $s \in\left[h_{0}, b_{0}\right], \theta\left(h_{0}\right)=0, \theta\left(b_{0}\right)=J\left(\delta_{0}\right) / \omega_{1}=1 / 2$, поэтому однозначно определена обратная функция $\theta^{-1} ;$ положим

$$
x_{0}(t):=\theta^{-1}(t), \quad t \in[0,1 / 2] .
$$

Распространим функцию $x_{0}(t)$ на весь отрезок $[0,1]$ симметричным образом, а именно положим

$$
x_{0}(t)=x_{0}(1-t), \quad t \in[1 / 2,1] .
$$

Очевидно, функция $x_{0}(t)$ является строго положительной, она монотонно возрастает при $t \in[0,1 / 2]$, причем $x_{0}(0)=h_{0}$ и $x_{0}(1 / 2)=b_{0}$. Таким образом, для всех значений $t \in[0,1]$ выполнены неравенства $0<h_{0} \leqslant x_{0}(t) \leqslant b_{0}$. Ниже будет показано, что функция $x_{0}(t)$ дважды дифференцируема на отрезке $[0,1]$ и при этом

$$
x_{0}^{\prime}(0)=x_{0}^{\prime}(1 / 2)=x_{0}^{\prime}(1)=0 .
$$

Нелинейная граничная задача второго порядка, которой удовлетворяет функция $x_{0}(t)$, записана ниже, в формуле (5.23), где нужно положить $\lambda=\lambda_{0}$. Также ниже при доказательстве леммы 10 будет показано, что $\left\|x_{0}\right\|_{p}=1$. Принимая во внимание $(5.8)$ и равенство $x_{0}(0)=h_{0}$, получаем формулу

$$
x_{0}(0)=x_{0}(1)=h_{0} .
$$

Таким образом, с учетом формул $(4.6),(5.9)$ и (5.10) заключаем, что функция $x_{0}(t)$ удовлетворяет на $[0,1]$ периодическим граничным условиям и $x_{0}(t) \in \operatorname{Ran}\left(A_{\eta}\right)$.

Продолжим периодически функцию $x_{0}(t)$ на всю ось $\mathbb{R}$ и полученную периодическую функцию с периодом 1 обозначим через $\hat{x}_{0}(t), t \in \mathbb{R}$.

Отметим следующее важное осцилляционное свойство функции $x_{0}(t)$. Функция $x_{0}(t)$ пересекает функцию $e_{0}(t) \equiv 1$ на полуоткрытом интервале $(0,1]$ ровно два 
раза - это минимально возможное число пересечений с функцией $e_{0}(t)$ для симметричной относительно точки $t=1 / 2$ положительной функции $x(t)$ при условии $\|x\|_{p}=1$. Заметим, что все функции из определенных ниже в (5.12) множеств $\mathcal{M}_{0}, \mathcal{M}_{1}$ пересекают функцию $e_{0}(t)$ на интервале $(0,1]$ ровно два раза (здесь и ниже мы говорим, что функции $f(t)$ и $g(t)$ пересекаются в точке $s \in(0,1]$, если выполнено равенство $f(s)=g(s))$.

Для фиксированного $p>p_{0}$ введем также функцию

$$
x_{1}(t):=h_{0}+b_{0}-x_{0}(t), \quad t \in[0,1] .
$$

Очевидно, что функция $x_{1}(t)$ является строго положительной симметричной относительно точки $t=1 / 2$ функцией. Используя формулы (5.9)-(5.11), убеждаемся, что справедливы равенства $x_{1}(0)=x_{1}(1)=b_{0}$ и $x_{1}^{\prime}(0)=x_{1}^{\prime}(1 / 2)=x_{1}^{\prime}(1)=0$. Таким образом, функция $x_{1}(t)$ также удовлетворяет на отрезке $[0,1]$ периодическим граничным условиям, и $x_{1}(t) \in \operatorname{Ran}\left(A_{\eta}\right)$. Несложно показать, что $\left\|x_{1}\right\|_{p}=1$.

Продолжим периодически функцию $x_{1}(t)$ на всю ось $\mathbb{R}$, полученную периодическую функцию с периодом 1 обозначим через $\hat{x}_{1}(t), t \in \mathbb{R}$.

Для числа $0 \leqslant \kappa \leqslant 1$ определим функции

$$
x_{0, \kappa}(t):=\hat{x}_{0}(t+\kappa), \quad x_{1, \kappa}(t):=\hat{x}_{1}(t+\kappa), \quad t \in[0,1] .
$$

Очевидно, $x_{0,0}(t) \equiv x_{0,1}(t) \equiv x_{0}(t), x_{1,0}(t) \equiv x_{1,1}(t) \equiv x_{1}(t)$. Кроме того, в силу определения и периодичности функций при всех $0 \leqslant \kappa \leqslant 1$ имеют место соотношения

$$
x_{0, \kappa}(t) \in \operatorname{Ran}\left(A_{\eta}\right), \quad\left\|x_{0, \kappa}\right\|_{p}=1, \quad x_{1, \kappa}(t) \in \operatorname{Ran}\left(A_{\eta}\right), \quad\left\|x_{1, \kappa}\right\|_{p}=1 .
$$

Введем два множества функций, заданных на [0,1]:

$$
\mathcal{M}_{0}:=\left\{x_{0, \kappa}(t): 0 \leqslant \kappa \leqslant 1\right\}, \quad \mathcal{M}_{1}:=\left\{x_{1, \kappa}(t): 0 \leqslant \kappa \leqslant 1\right\} .
$$

Отметим, что здесь элементы $x_{0,0}$ и $x_{0,1}$ отождествлены, так же отождествлены элементы $x_{1,0}$ и $x_{1,1}$, следовательно, многообразия $\mathcal{M}_{0}$ и $\mathcal{M}_{1}$ являются замкнутыми компактными кривыми, которые лежат в множестве $\operatorname{Ran}\left(A_{\eta}\right)$. Зададим два множества, центрально-симметричные множествам $\mathcal{M}_{0}$ и $\mathcal{M}_{1}$ :

$$
\mathcal{M}_{2}:=-\mathcal{M}_{0}=\left\{-x(t): x(t) \in M_{0}\right\}, \quad \mathcal{M}_{3}:=-\mathcal{M}_{1}=\left\{-x(t): x(t) \in M_{1}\right\} .
$$

ЛЕмма 10. Имеют место следующие утверждения.

1. Пусть фиксировано число $0<p \leqslant p_{0}$. Тогда минимум в экстремальной задаче

$$
\left\langle x, A_{\eta}^{-1} x\right\rangle \rightarrow \inf , \quad x \in D \cap \operatorname{Dom}\left(A_{\eta}^{-1}\right),
$$

равен $\omega_{1}^{2}$ и достигается на двух функииях $\pm e_{0}(t)$, лежащих на гранище дD. Минимум

$$
\inf _{x \in D \cap H_{A_{\eta}}}\|x\|_{A_{\eta}}^{2}
$$

также достигается только в двух точках $\pm e_{0}$. 
2. Пусть биксировано число $p_{0}<p<\infty$. Тогда минимум в экстремальной задаче (5.14) равен $\lambda_{0} p$ и достигается на одномерном компактном четырехсвязном многообразии

$$
\mathcal{M}:=\bigcup_{j=0}^{3} \mathcal{M}_{j} \subset \operatorname{Ran}\left(A_{\eta}\right),
$$

при этом компоненты $\mathcal{M}_{j}, j=0,1,2,3$, попарно не пересекаются и лежат на границе дD. Минимум (5.15) также достигается только на множестве $\mathcal{M}$.

ДокАЗАтЕЛьство. Покажем, что минимум в задаче (5.14) достижим. В силу леммы 8 максимальное собственное число оператора $A_{\eta}$ равно $\nu_{0}=1 / \omega_{1}^{2}$. Следовательно, минимальное собственное число оператора $A_{\eta}^{-1}$ равно $\omega_{1}^{2}$. Таким образом, согласно известному вариационному свойству собственных чисел компактного симметричного оператора [58], [59] справедлива оценка $\left\langle x, A_{\eta}^{-1} x\right\rangle \geqslant \omega_{1}^{2}\|x\|^{2}$ для $x \in L^{2}$. Из этой оценки и первой теоремы Вейерштрасса [56] следует достижимость минимума в задаче (5.14).

Решим экстремальную задачу (5.14) методом множителей Лагранжа [57]. Функция Лагранжа задается формулой $\mathcal{L}(x)=\lambda_{1}\left\langle x, A_{\eta}^{-1} x\right\rangle-\lambda_{2} G(x)$ для $x \in \operatorname{Ran}\left(A_{\eta}\right)$, где множители Лагранжа удовлетворяют неравенствам $\lambda_{1} \geqslant 0, \lambda_{2} \geqslant 0, \lambda_{1}^{2}+\lambda_{2}^{2}>0$. Используя формулы для первых двух производных квадратичного функционала [57] и равенства (5.2), (5.3), продифференцируем дважды функцию Лагранжа. Согласно принципу Лагранжа и условию второго порядка [57] заключаем, что для того чтобы точка $x \in \mathcal{X} \cap \operatorname{Ran}\left(A_{\eta}\right)$ доставляла минимум в задаче (5.14), необходимо выполнение условия стационарности

$$
\mathcal{L}^{\prime}(x) \equiv 2 \lambda_{1} A_{\eta}^{-1} x-\lambda_{2} G^{\prime}(x)=0,
$$

а также условия второго порядка

$$
\left\langle z, \mathcal{L}^{\prime \prime}(x) z\right\rangle \equiv\left\langle z,\left[2 \lambda_{1} A_{\eta}^{-1}-\lambda_{2} G^{\prime \prime}(x)\right] z\right\rangle \geqslant 0
$$

для всех $z$ таких, что $\left\langle z, G^{\prime}(x)\right\rangle=0$, и условия дополняющей нежесткости

$$
\lambda_{2}\left(\int_{0}^{1}|x(t)|^{p} d t-1\right)=0 .
$$

Легко проверить, что в нашей задаче $\lambda_{1}>0$ и $\lambda_{2}>0$, положим $\lambda=\lambda_{2} / 2 \lambda_{1}>0$. Учитывая формулы (5.3), (5.17)-(5.19), убеждаемся, что точка $x$, доставляющая минимум в задаче (5.14), является граничной точкой, и для нее выполнены соотношения $x=\lambda A_{\eta} G^{\prime}(x)$ (ср. с равенствами $\left.(2.2)\right)$ и

$$
\left\langle z,\left[A_{\eta}^{-1}-\lambda p(p-1)|x(t)|^{p-2}\right] z\right\rangle \geqslant 0, \quad \text { если }\left\langle z, G^{\prime}(x)\right\rangle=0 .
$$

Мы будем искать положительные решения, т. е. будем полагать, что $x(t)>0$ при $t \in[0,1]$; можно показать, что знакопеременные функции не доставляют минимума в экстремальной задаче (5.14).

Таким образом, уравнение для положительной экстремальной функции $x(t)$ согласно соотношениям (4.5) и (5.2) является нелинейным интегральным уравнением 
Хаммерштейна и имеет вид

$$
x(t)=\frac{\lambda p}{2 \omega_{1} \operatorname{sh} \frac{\omega_{1}}{2}} \int_{0}^{1} \operatorname{ch}\left(\omega_{1}|t-s|-\frac{\omega_{1}}{2}\right) x^{p-1}(s) d s, \quad t \in[0,1],
$$

где $\lambda>0$ - множитель Лагранжа, при этом выполнены условие второго порядка (5.20) и условие связи

$$
\int_{0}^{1} x^{p}(t) d t=1
$$

Поскольку $x \in \operatorname{Ran}\left(A_{\eta}\right)$, заключаем, учитывая (4.6), (4.7), что экстремальная функция дважды дифференцируема на отрезке $[0,1]$. Используя формулу для обратного оператора (4.7), убеждаемся, что интегральное уравнение (5.21) эквивалентно следующей нелинейной краевой задаче:

$$
\begin{gathered}
x^{\prime \prime}(t)=\omega_{1}^{2} x(t)-\lambda p x^{p-1}(t), \quad t \in[0,1], \\
x(0)=x(1), \quad x^{\prime}(0)=x^{\prime}(1) .
\end{gathered}
$$

Кроме того, учитывая вывод, сделанный в замечании 8, мы будем искать положительные решения задачи (5.22), (5.23), имеющие минимально возможное число пересечений с функцией $e_{0}(t) \equiv 1$ на интервале $(0,1]$. Как было указано выше, для симметричной положительной функции это минимальное число равно двум. Можно показать, что возможные положительные решения задачи (5.22), (5.23), пересекающие функцию $e_{0}(t)$ на интервале $(0,1]$ более двух раз, не доставляют минимум в задаче (5.14).

Записывая уравнение задачи (5.23) в виде $d\left(x^{\prime}(t)\right)^{2}=\omega_{1}^{2} d x^{2}(t)-2 \lambda d x^{p}(t)$, приходим к следующему факту: если функция $x(t)>0$ удовлетворяет краевой задаче (5.23), то эта функция удовлетворяет также следующей краевой задаче:

$$
\begin{gathered}
\left(x^{\prime}(t)\right)^{2}=\omega_{1}^{2} x^{2}(t)-2 \lambda x^{p}(t)+C, \quad t \in[0,1], \\
x(0)=x(1), \quad x^{\prime}(0)=x^{\prime}(1),
\end{gathered}
$$

при некоторой константе $C$. Детальный анализ задачи (5.24) с учетом соотношений $(5.20),(5.22)$ показывает, что имеются две возможности: $0<p<p_{0}$ и $p>p_{0}$.

Легко видеть, что для любого $p>0$ задача (5.22), (5.24) имеет положительное постоянное решение - функцию $e_{0}(t) \equiv 1$, заданную в (5.5). Подставляя $e_{0}(t)$ в $(5.23),(5.24)$, получаем следующие значения $\tilde{\lambda}_{0}, \widetilde{C}$ постоянных $\lambda, C$ в задаче $(5.24)$ :

$$
\tilde{\lambda}_{0}=\frac{\omega_{1}^{2}}{p}, \quad \widetilde{C}=\frac{2-p}{p} \omega_{1}^{2} .
$$

Условие второго порядка (5.20) показывает, что функция $e_{0}(t)$ является точкой локального минимума задачи (5.14) только при $0<p \leqslant p_{0}$.

В самом деле, согласно лемме 8, принимая во внимание вторую собственную функцию и второе собственное число и учитывая (5.25), получаем равенство

$$
\left\langle\varphi_{1},\left[A_{\eta}^{-1}-\tilde{\lambda}_{0} p(p-1) e_{0}^{p-2}\right] \varphi_{1}\right\rangle=(2-p) \omega_{1}^{2}+4 \pi^{2},
$$

выражение в правой части неотрицательно тогда и только тогда, когда $0<p \leqslant p_{0}$. Отметим, что в силу леммы 8 имеет место равенство $\left\langle\varphi_{1}, \varphi_{0}\right\rangle=\left\langle\varphi_{1}, e_{0}\right\rangle=0$ и, кроме 
того, $G^{\prime}\left(e_{0}\right)=p e_{0}$. Учитывая вышеизложенное, заключаем, что условие второго порядка (5.20) для функции $e_{0}$ не выполнено при $p>p_{0}$, и для этих значений $p$ точка $e_{0}$ не является точкой локального минимума задачи (5.14). Можно показать, что при $0<p \leqslant p_{0}$ функции $\pm e_{0}$ являются точками глобального минимума задачи (5.14). Используя формулы (4.7), (5.5), найдем минимальное значение:

$$
\left\langle e_{0}, A_{\eta}^{-1} e_{0}\right\rangle=\omega_{1}^{2} \int_{0}^{1} e_{0}^{2}(t) d t=\omega_{1}^{2} .
$$

Взяв гильбертово пространство $\left(H_{A_{\eta}},\|\cdot\|_{A_{\eta}}\right)$, ассоциированное с ковариационным оператором $A_{\eta}$, можно доказать, что минимум (5.15) также достигается только в двух точках $\pm e_{0}$. Первое утверждение леммы доказано.

Рассмотрим теперь вторую возможность. Покажем, что для $p>p_{0}$ задача (5.22), (5.24) имеет среди прочих два множества положительных решений - множество $\left\{e_{0}\right\}$, состоящее из единственной функции $e_{0}(t)$, и множество $\mathcal{M}_{0} \cup \mathcal{M}_{1}$; при этом минимум в задаче (5.14) доставляют точки из $\mathcal{M}_{0} \cup \mathcal{M}_{1}$. Множества $\mathcal{M}_{0}, \mathcal{M}_{1}$ целиком определяются только одной функцией $x_{0}(t)$, поэтому покажем сначала, что задача $(5.22),(5.24)$ имеет положительное решение $x_{0}(t)$.

Учитывая (5.23), несложно убедиться, что при $p>2$ допустимо обращение в ноль второй производной экстремали $x(t)$ во внутренней точке отрезка $[0,1]$. Принимая во внимание этот факт, а также отсутствие первой производной $x^{\prime}(t)$ в уравнении (5.23), заключаем, что задача $(5.22),(5.24)$ имеет симметричные решения, т. е. $x(t)=x(1-t)$ при $t \in[0,1]$. Всюду ниже мы полагаем, что $p>p_{0}$.

Итак, будем искать симметричные положительные решения задачи (5.22), (5.23), пересекающие функцию $e_{0}(t)$ на интервале $(0,1]$ ровно два раза, имея целью доказать, что среди таких решений присутствует функция $x_{0}(t)$.

Вследствие граничных условий в (5.23) заключаем, что симметричные экстремали удовлетворяют условиям $x^{\prime}(0)=x^{\prime}(1 / 2)=x^{\prime}(1)=0$. Пусть для определенности $x(0)=x(1)=h>0$.

Учитывая вышеизложенное, приходим к выводу, что симметричные экстремали удовлетворяют нелинейной граничной задаче первого порядка

$$
\begin{gathered}
\left(x^{\prime}(t)\right)^{2}=\omega_{1}^{2} x^{2}(t)-2 \lambda x^{p}(t)+C, \quad t \in[0,1 / 2], \\
x(0)=h, \quad x^{\prime}(0)=x^{\prime}(1 / 2)=0,
\end{gathered}
$$

где $h>0$ и $C$ - некоторые постоянные, уравнения для которых будут записаны ниже. Ниже также показано, что $C<0$.

Рассмотрим сначала случай, когда симметричная экстремаль возрастает в окрестности точки $t=0$. Исследуя задачу (5.26) в этом случае и учитывая, что мы ищем экстремаль, пересекающую функцию $e_{0}(t)$ на интервале $(0,1]$ ровно два раза, несложно убедиться, что искомая симметричная экстремаль монотонно возрастает на всем отрезке $[0,1 / 2]$ от значения $h=x(0)$ до значения $b:=x(1 / 2)$, при этом экстремаль является сначала выпуклой вниз, затем - выпуклой верх и удовлетворяет нелинейной граничной задаче

$$
\begin{gathered}
x^{\prime}(t)=\sqrt{\omega_{1}^{2} x^{2}(t)-2 \lambda x^{p}(t)+C}, \quad t \in[0,1 / 2], \\
x(0)=h, \quad x^{\prime}(0)=x^{\prime}(1 / 2)=0 .
\end{gathered}
$$


Подставляя в (5.26) значения $t=0$ и $t=1 / 2$, получаем два уравнения для параметров $0<h<b, \lambda>0$ и $C$ :

$$
\begin{gathered}
\omega_{1}^{2} h^{2}-2 \lambda h^{p}+C=0 \\
\omega_{1}^{2} b^{2}-2 \lambda b^{p}+C=0 .
\end{gathered}
$$

Ниже в лемме 11 доказано, что уравнение

$$
\omega_{1}^{2} x^{2}-2 \lambda x^{p}+C=0, \quad x>0,
$$

имеет при $p>p_{0}$ ровно одну пару решений, удовлетворяющих условию $x<b$, если $c_{0}<C<0$, где число $c_{0}<0$ указано в лемме 11. Далее, интегрируя уравнение (5.27), заключаем, что искомая экстремаль $\tilde{x}_{0}(t)$ удовлетворяет уравнению

$$
\int_{0}^{x(t)} \frac{d x}{\sqrt{\omega_{1}^{2} x^{2}-2 \lambda x^{p}+C}}=t, \quad t \in[0,1 / 2] .
$$

Отсюда следует, что, как и указано выше, $h>0$. В самом деле, если $h=0$, то в силу (5.28) получаем $C=0$, и интеграл в левой части соотношения (5.31) расходится в нуле, что противоречит конечности правой части уравнения (5.31).

Покажем, что при определенном выборе параметров $h, b, \lambda$ и $C$ функция $\tilde{x}_{0}(t)$ совпадает с функцией $x_{0}(t)$, введенной в $(5.7),(5.8)$.

Определим монотонно возрастающую функцию

$$
\tilde{\theta}(s):=\int_{0}^{s} \frac{d x}{\sqrt{\omega_{1}^{2} x^{2}-2 \lambda x^{p}+C}}, \quad 0 \leqslant s \leqslant b .
$$

Тогда, как легко видеть,

$$
\tilde{x}_{0}(t)=\tilde{\theta}^{-1}(t), \quad t \in[0,1 / 2] .
$$

Теперь выпишем уравнения для параметров $h, b, \lambda$. Из уравнений (5.28), (5.29) получаем

$$
\omega_{1}^{2} h^{2}-2 \lambda h^{p}=\omega_{1}^{2} b^{2}-2 \lambda b^{p} .
$$

Положив в (5.31) $t=1 / 2$, находим

$$
\int_{0}^{b} \frac{d x}{\sqrt{\omega_{1}^{2} x^{2}-2 \lambda x^{p}+C}}=\frac{1}{2} .
$$

Используя условие нормировки (5.22), симметричность функции $\tilde{x}_{0}(t)$ и формулу (5.33), получаем

$$
1=2 \int_{0}^{1 / 2} \tilde{x}^{p}(t) d t=2 \int_{0}^{1 / 2}\left[\tilde{\theta}^{-1}(t)\right]^{p} d t=2 \int_{0}^{b} \frac{s^{p} d s}{\sqrt{\omega_{1}^{2} s^{2}-2 \lambda s^{p}+C}}
$$

здесь во втором интеграле была проведена замена переменной $t=\tilde{\theta}(s)$. Покажем, что имеет место следующее утверждение. 
ПреДЛОЖЕНИЕ 4. Уравнения (5.34)-(5.36) относителъно параметров $h, b, \lambda$ имеют единственное решение - тройку $h_{0}, b_{0}, \lambda_{0}$, определенную в (1.8), (1.10)

ДокАЗАтЕЛьство. Учитывая формулу (5.34) и вводя параметр $\delta:=h / b \in(0,1)$, мы можем записать интеграл в левой части (5.35) в виде

$$
\int_{0}^{b} \frac{d x}{\sqrt{\omega_{1}^{2} x^{2}-2 \lambda x^{p}+C}}=\frac{1}{\omega_{1}} \int_{0}^{1} \frac{d x}{\sqrt{\left(2 \lambda / \omega_{1}^{2}\right) b^{p-2}\left(1-x^{p}\right)-\left(1-x^{2}\right)}}=\frac{1}{\omega_{1}} J(\delta)
$$

здесь во втором интеграле была проведена замена переменной $t=x / b$ и использована формула (1.3). Аналогично, последний интеграл в (5.36) можно записать каK

$$
\int_{0}^{b} \frac{s^{p} d s}{\sqrt{\omega_{1}^{2} s^{2}-2 \lambda s^{p}+C}}=\frac{b^{p}}{\omega_{1}} M(\delta),
$$

где $M(\delta)$ определено в (1.4). Из формул (5.34)-(5.38) получаем следующую систему из трех уравнений для трех параметров $\delta, b, \lambda$ :

$$
J(\delta)=\frac{\omega_{1}}{2}, \quad M(\delta)=\frac{\omega_{1}}{2 b^{p}}, \quad \lambda=\omega_{1}^{2} \frac{1-\delta^{2}}{\left(1-\delta^{p}\right) b^{p-2}} .
$$

Согласно лемме 1 первое уравнение в (5.39) имеет единственный корень $\delta_{0} \in(0,1)$. Зная $\delta_{0}$, из второго уравнения в (5.39) находим единственное решение $b_{0}$, заданное в (1.8). Из третьего уравнения в (5.39) находим единственное решение $\lambda_{0}$, заданное в формуле (1.10). Далее, согласно определениям получаем $h_{0}=b_{0} \delta_{0}$ и $C_{0}=2 \lambda_{0} b_{0}^{p}-$ $\omega_{1}^{2} b_{0}^{2}$. Утверждение доказано.

Используя формулы (5.6) и (5.32), убеждаемся, что при $C=C_{0}, h=h_{0}, b=b_{0}$, $\lambda=\lambda_{0}$ функции $\tilde{\theta}(s)$ и $\theta(s)$ тождественно равны; следовательно, при указанных значениях параметров, тождественно равны и функции $\tilde{x}_{0}(t)$ и $x_{0}(t)$.

Учитывая вышеизложенное, мы заключаем, что если симметричная экстремаль возрастает в окрестности точки $t=0$, то задача $(5.22),(5.24)$ имеет положительное симметричное решение $x_{0}(t)$.

Рассмотрим теперь случай, когда симметричная экстремаль убывает в окрестности точки $t=0$.

Рассуждения, аналогичные проведенным в предыдущем случае, доказывают, что теперь задача (5.22), (5.24) имеет в качестве положительного симметричного решения (пересекающего функцию $e_{0}(t)$ на интервале $(0,1]$ ровно два раза) функцию $x_{1}(t)$, заданную в $(5.11)$. В частности, функция $x_{1}(t)$ монотонно убывает на всем отрезке $[0,1 / 2]$ от значения $x_{1}(0)=b_{0}$ до значения $x_{1}(1 / 2)=h_{0}$, при этом она является сначала выпуклой вверх, затем - выпуклой вниз и удовлетворяет следующей нелинейной граничной задаче:

$$
\begin{gathered}
x^{\prime}(t)=-\sqrt{\omega_{1}^{2} x^{2}(t)-2 \lambda_{0} x^{p}(t)+C_{0}}, \quad t \in[0,1 / 2], \\
x(0)=b_{0}, \quad x^{\prime}(0)=x^{\prime}(1 / 2)=0,
\end{gathered}
$$

где $h_{0}, b_{0}, \lambda_{0}, C_{0}$ те же, что и выше. 
Вывод. Учитывая доказанные утверждения о функциях $x_{0}(t), x_{1}(t)$ и используя определения $(5.12),(5.13)$ и свойства периодичности, несложно убедиться, что при $p>p_{0}$ задача $(5.22),(5.24)$ имеет наряду с функцией $e_{0}$ множество положительных решений $\mathcal{M}_{0} \cup \mathcal{M}_{1}$ и множество отрицательных решений $-e_{0}, \mathcal{M}_{1} \cup \mathcal{M}_{2}$. Как уже было сказано, при $p>p_{0}$ точка $e_{0}$ не является точкой локального минимума.

Можно показать, что при $p>p_{0}$ задача (5.22), (5.24), кроме функций из множества $\mathcal{M}_{0} \cup \mathcal{M}_{1}$, не имеет иных положительных решений, пересекающих функцию $e_{0}(t)$ на интервале $(0,1]$ ровно два раза.

Используя формулы (4.7), (5.22), (5.23), получаем что для любого $z \in \mathcal{M}_{0} \cup \mathcal{M}_{1}$

$$
\left\langle z, A_{\eta}^{-1} z\right\rangle=\left\langle x_{0}, A_{\eta}^{-1} x_{0}\right\rangle=p \lambda_{0} .
$$

Из последнего утверждения, формулы (5.40) и того факта, что минимум в экстремальной задаче (5.14) доставляют только знакопостоянные функции, вытекает, что при $p>p_{0}$ минимум в задаче (5.14) равен $\lambda_{0} p$ и достигается на одномерном компактном многообразии $\mathcal{M}$ из (5.16).

Можно также показать, используя хорошие свойства дифференцируемости границы $\partial D$, что при $p>p_{0}$ минимум (5.15) также достигается только на множестве $\mathcal{M}$. Кроме того, легко видеть, что компоненты $\mathcal{M}_{j}, j=0,1,2,3$, взаимно не пересекаются и лежат на границе $\partial D$. Таким образом, для завершения доказательства второго утверждения леммы 10 нам осталось доказать леммы 1 и 11. Это сделано ниже.

Для $x>0$ введем функцию $q(x):=2 \lambda x^{p}-\omega_{1}^{2} x^{2}$. Производная функции $q(x)$ имеет вид $q^{\prime}(x)=2 x\left(\lambda p x^{p-2}-\omega_{1}^{2}\right), x>0$. Используя две последние формулы, заключаем, что $q(0)=0, q(+\infty)=+\infty$, функция $q(x)$ имеет при $x>0$ один глобальный минимум в точке $x_{\min }=\left(\omega_{1}^{2} / \lambda p\right)^{1 /(p-2)}$, монотонно убывает на интервале $\left(0, x_{\min }\right)$ и монотонно возрастает на интервале $\left(x_{\min }, \infty\right)$.

ЛЕмма 11. Для фиксированнъх $\omega_{1}>0, \lambda>0, C \in \mathbb{R}$ уравнение

$$
\omega_{1}^{2} x^{2}-2 \lambda x^{p}+C=0, \quad x>0,
$$

имеет при $p>p_{0}$ ровно одну пару решений, удовлетворяющих условию $0<x<b$, если $c_{0}:=q\left(x_{\min }\right)<C<0$.

ДокАЗАтЕльство. Запишем уравнение (5.41) в виде

$$
q(x)=C, \quad x>0,
$$

и решим его, применяя графический метод. Нарисуем в обычной декартовой системе координат $O x y$ графики двух функций $y=q(x)$ и $y=C$. Из чертежа и свойств функции $q(x)$ следует, что уравнение (5.42) имеет ровно два решения $0<x_{1}=h<x_{2}=b$, если $q\left(x_{\min }\right)<C<0$. Лемма доказана.

ДокАЗАТЕЛЬСтво ЛЕммы 1. Записывая формулу (1.6) при $p=p_{0}$, получаем равенство

$$
J_{p_{0}}(1)=\frac{\pi}{\sqrt{p_{0}-2}}=\frac{\omega_{1}}{2} .
$$

Рассуждая так же, как при доказательстве леммы 11, и используя (5.43), заключаем, что уравнение

$$
J_{p_{0}}(\delta)=\frac{\omega_{1}}{2}, \quad 0<\delta \leqslant 1,
$$


имеет ровно один корень $\delta_{0}\left(p_{0}, \omega_{1}\right)=1$; здесь $x_{1}=h=x_{2}=b$. Учитывая этот факт и свойство монотонного убывания по $p$ функции $J_{p}(\delta)$ из леммы 4 , получаем утверждение леммы 1. Доказательство леммы 10 завершено.

ЗАмечание 9. Отметим, что в утверждениях леммы 10 имеет место свойство непрерывности по параметру $p$ при $p \downarrow p_{0}$, в том числе непрерывность для минимальных значений. Так, можно доказать, что

$$
\lim _{p \downarrow p_{0}} p \lambda_{0}\left(p, \omega_{1}\right)=\omega_{1}^{2} .
$$

Свойство непрерывности по параметру $p$ при $p \uparrow \infty$ рассмотрено ниже в п. 5.3.

ЗАмЕчАНИЕ 10. При $p=2$ первое утверждение леммы 10 согласуется с леммой 8: функция (5.5) при $p=2$ совпадает с собственной функцией $\varphi_{0}(t)$ из формулы (4.9), а минимальное значение $\omega_{1}^{2}$ совпадает с $1 / \nu_{0}$.

ЗАмечание 11. Можно показать, что для достаточно больших $p>p_{0}$ задача $(5.22),(5.24)$ имеет множество положительных решений, пересекающих функцию $e_{0}(t)$ на интервале $(0,1]$ ровно 4,6 и т. д. раз.

5.3. Сравнение со случаем $p=\infty$. В дополнение к замечанию 9 укажем, что в утверждении леммы 10 имеет место свойство непрерывности по параметру $p$ также при $p \uparrow \infty$, в том числе для минимальных значений. Для описания этого свойства нам понадобятся некоторые определения и утверждения, которые мы приведем ниже.

Согласно формуле (3.8) определена функция

$$
J_{\infty}(\delta)=\ln \frac{1+\sqrt{1-\delta^{2}}}{\delta} .
$$

В соответствии с уравнением (1.7) обозначим через $\delta_{\infty} \equiv \delta_{\infty}\left(\omega_{1}\right)$ единственный корень уравнения

$$
J_{\infty}(\delta)=\frac{\omega_{1}}{2}, \quad 0<\delta<1 .
$$

Используя (5.45), легко находим, что $\delta_{\infty}=1 / \operatorname{ch}\left(\omega_{1} / 2\right)$, и это согласуется с последней формулой в (1.8).

ЛЕмма 12. Для любого фиксированного $\omega_{1}>0$ функиия $\delta_{0}\left(p, \omega_{1}\right)$ является строго монотонно убывающей функиией переменной $p \in\left(p_{0}, \infty\right)$. Существует пре$\partial е л$

$$
\delta_{0}\left(\infty, \omega_{1}\right):=\lim _{p \uparrow \infty} \delta_{0}\left(p, \omega_{1}\right)=\delta_{\infty}=\frac{1}{\operatorname{ch} \frac{\omega_{1}}{2}} .
$$

ДокаЗАтЕЛЬство. Монотонность функции $\delta_{0}\left(p, \omega_{1}\right)$ вытекает непосредственно из леммы 6 и определения функции $\delta_{0}\left(p, \omega_{1}\right)$. Монотонно убывающая и ограниченная последовательность имеет предел, отсюда следует существование предела в (5.47). Второе равенство в (5.47) получаем, используя определение числа $\delta_{\infty}$ посредством формул $(5.45),(5.47)$.

Из этой леммы, очевидно, вытекают следующие неравенства: для любых $p>p_{0}$ и $\omega_{1}>0$

$$
0<\delta_{\infty}\left(\omega_{1}\right)<\delta_{0}\left(p, \omega_{1}\right)<h_{0}\left(p, \omega_{1}\right)<1<b_{0}\left(p, \omega_{1}\right) .
$$

Таким образом, доказано второе неравенство в (1.9). 
ЛЕмма 13. Для любого биксированного $\omega_{1}>0$ бункиия $p \lambda_{0}\left(p, \omega_{1}\right)$ монотонно убывает по $p \in\left(p_{0}, \infty\right)$ от значения $p_{0} \lambda_{0}\left(p_{0}, \omega_{1}\right)=\omega_{1}^{2}$ до значения

$$
\lim _{p \uparrow \infty} p \lambda_{0}\left(p, \omega_{1}\right)=2 \omega_{1} \text { th } \frac{\omega_{1}}{2} .
$$

ДокАЗАТЕЛЬСтво. Согласно второму утверждению леммы 10 при $p>p_{0}$ справедливо равенство

$$
\min _{x \in D \cap \operatorname{Dom}\left(A_{\eta}^{-1}\right)}\left\langle x, A_{\eta}^{-1} x\right\rangle=p \lambda_{0}\left(p, \omega_{1}\right) .
$$

Используя неравенство (1.51) при $\beta=1$ и равенство (5.1), убеждаемся, что выражение в левой части формулы (5.48) является убывающей функцией по $p \in\left(p_{0}, \infty\right)$. Остальные утверждения леммы вытекают из формулы (5.44) и приведенного ниже соотношения (5.49). Лемма доказана.

ЛЕмма 14. Справедливо соотношение

$$
\lim _{p \uparrow \infty} p \lambda_{0}\left(p, \omega_{1}\right)=2 \omega_{1} \sqrt{1-\delta_{\infty}^{2}}=2 \omega_{1} \text { th } \frac{\omega_{1}}{2} .
$$

ДокАЗАТЕЛЬСТво является довольно длинным и техническим, мы его опускаем.

5.4. Завершение доказательств п. 3 и п. 4 теоремы 2. Дальнейшее доказательство п. 3 теоремы 2 проводится так же, как доказательство п. 3 теоремы 3 из статьи [30], с учетом леммы 6 , первого утверждения леммы 10 и условия $2<p<p_{0}$.

Докажем п. 4 теоремы 2 , используя второе утверждение теоремы 9 , где мы положим $E=L^{2}[0,1], P_{A}=\mu_{\eta}, A=A_{\eta}$. Как уже было сказано, множество $D$ из (5.4) удовлетворяет условию $\mathbf{C 1}$ при $p>p_{0}$, и поэтому для него выполнено соотношение (2.4). Применяя это соотношение с учетом замечания 6 , формулы (5.1) и второго утверждения леммы 10, получаем следующую цепочку равенств при $p>p_{0}$ :

$$
\begin{aligned}
\lim _{v \rightarrow \infty} \frac{1}{v^{2}} \ln \mu_{\eta}(v D) & =\lim _{v \rightarrow \infty} \frac{1}{v^{2}} \ln \mathbf{P}\left\{\int_{0}^{1}|\eta(t)|^{p} d t>v^{p}\right\}= \\
& =-\inf _{x \in D} I_{A_{\eta}}(x)=-\frac{1}{2} \inf _{x \in D \cap \operatorname{Ran}\left(A_{\eta}\right)}\left\langle x, A_{\eta}^{-1} x\right\rangle=-\frac{\lambda_{0} p}{2} .
\end{aligned}
$$

Полагая $v=u \sqrt{m} / \beta^{(2+p) / 2 p}$ и используя (4.4), получаем соотношение (1.25). Четвертое утверждение теоремы 2 доказано.

\section{6. ДОКАЗАТЕЛЬСТВО П. 3 И П. 4 ТЕОРЕМЫ 1}

Мы докажем п. 3 и п. 4 теоремы 1, применяя теоремы 7 и 9 . Некоторые утверждения ниже мы формулируем в достаточно общем виде, рассматривая случай $p>0$ и имея целью обоснование соотношения (1.26) из замечания 1.

Используем масштабное свойство процесса Боголюбова из предложения 3 для интегралов из теоремы 1. Имеет место следующее утверждение.

Лемма 15. Для любых $p>0,0<\alpha<2, u>0$ выполнено равенство

$$
\mathbf{E} \exp \left\{u\left(\int_{0}^{\beta}|\xi(t)|^{p} d t\right)^{\alpha / p}\right\}=\mathbf{E} \exp \left\{r\left(\int_{0}^{1}|\eta(s)|^{p} d s\right)^{\alpha / p}\right\}, \quad r=u \frac{\beta^{\alpha(2+p) / 2 p}}{m^{\alpha / 2}} .
$$


ДокАЗАТЕЛьство. Сделав в первом интеграле в (6.1) замену переменной $t=\beta s$ и используя формулу (4.1), получаем равенство (6.1).

Таким образом, в силу только что доказанной леммы нам достаточно доказать вариант теоремы 1 для процесса Боголюбова $\eta(t)$ с параметрами $\left(1,1, \omega_{1}\right)$ и большим параметром $r \rightarrow \infty$.

6.1. Выбор банахова функционального пространства. Зафиксируем числа $p>0$ и $0<\alpha<2$. Положим

$$
\tilde{p}:=\left\{\begin{array}{lll}
p, & \text { если } & p>2, \\
2, & \text { если } 0<p \leqslant 2 .
\end{array}\right.
$$

Учитывая лемму 15, возьмем в качестве банахова пространства $E$ из теорем 7 и 9 банахово пространство $L^{\tilde{p}} \equiv L^{\tilde{p}}[0,1]$ функций, интегрируемых в $\tilde{p}$-й степени на отрезке $[0,1]$. Норма в $L^{\tilde{p}}$ обозначена через $\|\cdot\|_{\tilde{p}}$.

Таким образом, в силу (6.2) мы имеем $\tilde{p} \geqslant 2$. Пусть $2 \geqslant \tilde{q}>1$ обозначает число, сопряженное к $\tilde{p}$, т. е. $1 / \tilde{p}+1 / \tilde{q}=1$. Напомним что, сопряженным банаховым пространством к пространству $L^{\tilde{p}}$ является пространство $L^{\tilde{q}}$.

В настоящем разделе мы будем рассматривать гауссовскую меру Боголюбова $\mu_{\eta}$, заданную в пространстве $L^{\tilde{p}}[0,1]$, имея в виду, что эта мера сосредоточена на $C^{0}[0,1]$. Известно, что в этом случае мера $\mu_{\eta}$ имеет нулевое среднее значение и инъективный ковариационный оператор $A_{\eta}: L^{\tilde{q}} \rightarrow L^{\tilde{p}}$ интегрального типа, который определяется по формуле (4.5), где $x \in L^{\tilde{q}}$. Здесь также справедлива лемма 7. Отметим, что теперь оператор $A_{\eta}$ зависит от $\tilde{p}$, в частности $\operatorname{Dom}\left(A_{\eta}\right)=L^{\tilde{q}}$, указывать эту зависимость мы не будем.

В настоящем разделе мы будем обозначать через $\langle\cdot, \cdot\rangle$ каноническую билинейную форму между пространствами $L^{\tilde{p}}$ и $L^{\tilde{q}}$, которая, как известно, имеет интегральный вид:

$$
\langle h, g\rangle=\int_{0}^{1} h(t) g(t) d t, \quad h \in L^{\tilde{p}}, g \in L^{\tilde{q}} .
$$

6.2. Выбор основного функционала. Мы докажем теорему 1 , применяя теоремы 7 и 9 , где мы будем полагать $E=L^{\tilde{p}}, P_{A}=\mu_{\eta}, A=A_{\eta}$ и $f \equiv 1$.

Возьмем в качестве функционала $F$, участвующего в формулировке теорем 7 и 9 , следующий функционал:

$$
\Psi(x):=-\|x\|_{p}^{\alpha} \equiv-\left(\int_{0}^{1}|x(t)|^{p} d t\right)^{\alpha / p}, \quad x \in L^{\tilde{p}} .
$$

Наша цель - применить теоремы 7 и 9 к этому функционалу и гауссовской мере Боголюбова $\mu_{\eta}$. Положим

$$
v=r^{1 /(2-\alpha)}=u^{1 /(2-\alpha)} \frac{\beta^{\alpha(2+p) /(2 p(2-\alpha))}}{m^{\alpha /(2(2-\alpha))}} .
$$

Тогда $r=v^{2-\alpha}$, кроме того, $v \rightarrow \infty$ при $r \rightarrow \infty$. Функционал (6.3) обладает свойством однородности порядка $\alpha: \Psi(c x)=c^{\alpha} \Psi(x), c>0$. Используя это свойство и 
равенство (6.4), запишем среднее значение из правой части формулы (6.1) в виде модельного интеграла Лапласа:

$$
\mathbf{E} \exp \left\{r\left(\int_{0}^{1}|\eta(t)|^{p} d t\right)^{\alpha / p}\right\}=\mathbf{E} e^{-r \Psi(\eta)}=\int_{L^{\tilde{p}}} e^{-v^{2} \Psi(x)} d \mu_{\eta}(v x) .
$$

Функционал $-\Psi(x)$ представляет собой степень интегрального функционала. Как известно, интегральные функционалы дифференцируемы по Фреше [42], [56], [57]. Напомним, что множество $\mathcal{X}$ было определено перед леммой 9.

ЛЕмма 16. Имеют место следующие утверждения.

1. Функиионал $\Psi$ трижды дифферениируем по Фреше в каждой точке $x \in \mathcal{X}$, при этом первая производная имеет вид

$$
\Psi^{\prime}(x)=-\alpha\left(\int_{0}^{1}|x(s)|^{p} d s\right)^{(\alpha-p) / p}|x(t)|^{p-1} \operatorname{sign} x(t),
$$

а вторую производную $\Psi^{\prime \prime}(x)$ можно рассматривать как линейный оператор $\Psi^{\prime \prime}(x): L^{\tilde{p}} \rightarrow L^{\tilde{q}}$, действующий по правилу

$$
\begin{aligned}
\Psi^{\prime \prime}(x) g= & \alpha(1-p)\|x\|_{p}^{\alpha-p}|x(t)|^{p-2} g+ \\
& +\alpha(p-\alpha)\|x\|_{p}^{\alpha-2 p}|x(t)|^{p-1} \operatorname{sign} x(t)\left\langle g,|x(t)|^{p-1} \operatorname{sign} x(t)\right\rangle, \quad g \in L^{\tilde{p}} .
\end{aligned}
$$

2. При $p \geqslant 2$ производные (6.6), (6.7) задают ограниченные линейные операторь при $x \in C^{0}[0,1]$.

ДокАЗАтЕльство. Утверждение леммы получаем, применяя теорему о суперпозиции дифференцируемых отображений [57] и известные формулы для первых двух производных функционала $G_{0}(x)=\int_{0}^{1}|x(t)|^{p} d t$ (см. лемму 9 и книги [42], [57]).

Лемма 17. Для функиионала $\Psi$ выполнено условие D1.

ДоКАЗАТЕЛЬСТВо проводится при помощи неравенства (1.51) с $\beta=1$.

6.3. Решение экстремальной задачи. Решим экстремальную задачу из условия D2 для функционала (6.3) и ковариационного оператора $A_{\eta}$, заданного в $(4.5)$ с $x \in L^{\tilde{q}}$. Введем следующие величины:

$$
\begin{aligned}
& c_{1}=c_{1}(\alpha)=\left(\frac{\alpha}{\omega_{1}^{2}}\right)^{1 /(2-\alpha)}, \quad e_{1}(t):=c_{1} e_{0}(t) \equiv c_{1}, \quad t \in[0,1], \\
& c_{0}=c_{0}(p, \alpha)=\left(\frac{\alpha}{\lambda_{0} p}\right)^{1 /(2-\alpha)}, \quad p>p_{0},
\end{aligned}
$$

где числа $p_{0}, \lambda_{0}$ заданы в формулах $(1.2),(1.10)$, а функция $e_{0}(t)$ - в формуле $(5.5)$. Положим

$$
\begin{aligned}
& \tilde{q}_{0}=\tilde{q}_{0}(p, \alpha)=\frac{2-\alpha}{2}\left(\frac{\alpha}{\omega_{1}^{2}}\right)^{\alpha /(2-\alpha)}>0, \\
& \tilde{q}_{1}=\tilde{q}_{1}(p, \alpha)=\frac{2-\alpha}{2}\left(\frac{\alpha}{\lambda_{0} p}\right)^{\alpha /(2-\alpha)}>0 .
\end{aligned}
$$


Отметим, что число $\tilde{q}_{0}$ получается из числа $q_{0}$, заданного в $(1.13)$, если в (1.13) положить $m=1, \beta=1, \omega=\omega_{1}$. Аналогичным образом число $\tilde{q}_{1}$ получается из числа $q_{1}$, заданного в (1.18). Эти два факта согласуются со связью между процессами $\xi(t)$ и $\eta(t)$, описанной в предложении 3 .

ЛЕмма 18. Имеют место следующие утверждения.

1. Пусть фиксировань числа $0<p \leqslant p_{0} u 0<\alpha<2$. Тогда минимум в экстремальной задаче

$$
\Lambda(x) \equiv \frac{1}{2}\left\langle x, A_{\eta}^{-1} x\right\rangle+\Psi(x) \rightarrow \inf , \quad x \in \operatorname{Dom}\left(A_{\eta}^{-1}\right),
$$

равен $-\tilde{q}_{0}$ и достигается на двух функииях $\pm e_{1}(t)$. При этом минимум

$$
\inf _{x \in H_{A_{\eta}}}\left[\Psi(x)+\frac{1}{2}\|x\|_{A_{\eta}}^{2}\right]
$$

также достигается только в двух точках $\pm e_{1}(t)$.

2. Пусть фиксированы числа $p>p_{0} u 0<\alpha<2$. Тогда минимум в экстремальной задаче (6.12) равен - $\tilde{q}_{1}$ и достигается на одномерном компактном четырехсвязном многообразии

$$
\mathcal{S}:=c_{0} \mathcal{M}=\left\{c_{0} x: x \in \mathcal{M}\right\} \subset \operatorname{Ran}\left(A_{\eta}\right),
$$

где $\mathcal{M}$ задано в формуле (5.16). Минимум (6.13) такюе достигается только на множестве $\mathcal{S}$.

ДокАЗАТЕЛЬСТво. Вывод обоих утверждений леммы основан на лемме 10 и проводится единым образом. Переходя к условной экстремальной задаче, убеждаемся, что справедливы следующие равенства:

$$
\begin{aligned}
T & :=\inf _{x \in \operatorname{Dom}\left(A_{\eta}^{-1}\right)}\left[\frac{1}{2}\left\langle x, A_{\eta}^{-1} x\right\rangle-\|x\|_{p}^{\alpha}\right]=\inf _{c>0} \inf _{\substack{x \in \operatorname{Dom}^{\prime}\left(A_{\eta}^{-1}\right) \\
\|x\|_{p}=c}}\left[\frac{1}{2}\left\langle x, A_{\eta}^{-1} x\right\rangle-\|x\|_{p}^{\alpha}\right]= \\
& =\inf _{c>0} \inf _{\substack{x \in \operatorname{Dom}^{\prime}\left(A_{\eta}^{-1}\right) \\
\|x\|_{p}=c}}\left[\frac{1}{2}\left\langle x, A_{\eta}^{-1} x\right\rangle-c^{\alpha}\right] .
\end{aligned}
$$

Решим внутреннюю экстремальную задачу в последнем выражении в (6.15). Согласно лемме 10 при фиксированном $c>0$ имеют место равенства

$$
\inf _{\substack{x \in \operatorname{Dom}\left(A_{\eta}^{-1}\right) \\\|x\|_{p}=c}} \frac{1}{2}\left\langle x, A_{\eta}^{-1} x\right\rangle=\inf _{\substack{z \in \operatorname{Dom}\left(A_{\eta}^{-1}\right) \\\|z\|_{p}=1}} \frac{c^{2}}{2}\left\langle z, A_{\eta}^{-1} z\right\rangle=\frac{c^{2}}{2 \sigma^{2}},
$$

где:

1) при $0<p \leqslant p_{0}$ минимум в первой экстремальной задаче в (6.16) достигается на двух функциях $\pm x^{c}(t):= \pm c e_{0}(t)$, а $\sigma^{2}=\sigma_{1}^{2}:=1 / \omega_{1}^{2}$;

2) при $p>p_{0}$ минимум в первой экстремальной задаче в (6.16) достигается на одномерном многообразии $\mathcal{S}_{c}:=c \mathcal{M}=\{c x: x \in \mathcal{M}\} \subset \operatorname{Ran}\left(A_{\eta}\right)$ и $\sigma^{2}=\sigma_{0}^{2}:=1 / \lambda_{0} p$. 
Таким образом, из соотношений (6.15), (6.16) получаем равенства

$$
T=\inf _{c>0}\left(\frac{c^{2}}{2 \sigma^{2}}-c^{\alpha}\right)=\frac{c_{\min }^{2}}{2 \sigma^{2}}-c_{\min }^{\alpha}=-\frac{2-\alpha}{2}\left(\alpha \sigma^{2}\right)^{\alpha /(2-\alpha)},
$$

где $c_{\min }=\left(\alpha \sigma^{2}\right)^{1 /(2-\alpha)}$. Используя формулы $(6.8)-(6.11)$ и (6.14)-(6.17), убеждаемся, что при $0<p \leqslant p_{0}$ мы имеем $c_{\text {min }}=c_{1}, T=-\tilde{q}_{0}$, а при $p>p_{0}$ выполнены равенства $c_{\min }=c_{0}, T=-\tilde{q}_{1}, \mathcal{S}_{c_{0}}=\mathcal{S}$. Отсюда с учетом хороших свойств гильбертова пространства $H_{A_{\eta}}$ и свойства гладкости функционала $\Psi$ вытекают оба утверждения леммы.

6.4. Завершение доказательств п. 3 и п. 4 теоремы 1. Дальнейшее доказательство п. 3 теоремы 1 проводится так же, как доказательство п. 3 теоремы 1 из статьи [30] с учетом формул (6.1), (6.4), (6.5), утверждения 1 леммы 18 и условия $2<p<p_{0}$.

Докажем п. 4 теоремы 1 при $p>p_{0}$, используя утверждение 3 теоремы 9, где мы положим $E=L^{p}[0,1], P_{A}=\mu_{\eta}, A=A_{\eta}, F=\Psi$. Как указано в лемме 17 , функционал $\Psi$ из формулы (6.3) удовлетворяет условию D1, поэтому для него выполнено соотношение (2.5). Применяя его с учетом замечания 7, формул (6.1), (6.3), (6.5), (6.11) и п. 2 леммы 18, получаем цепочку равенств при $p>p_{0}$ и $u \rightarrow \infty$ :

$$
\begin{aligned}
& \ln \mathbf{E} \exp \left\{u\left(\int_{0}^{\beta}|\xi(t)|^{p} d t\right)^{\alpha / p}\right\}=\ln \int_{L^{p}} e^{-v^{2} \Psi(x)} d \mu_{\eta}(v x)= \\
& =-\inf _{x \in L^{p}}\left[\Psi(x)+I_{A_{\eta}}(x)\right] v^{2}(1+o(1))=\tilde{q}_{1} v^{2}(1+o(1))= \\
& =\frac{2-\alpha}{2}\left(\frac{\alpha}{\lambda_{0} p}\right)^{\alpha /(2-\alpha)} v^{2}(1+o(1))=\frac{2-\alpha}{2}\left(\frac{\alpha \beta^{(2+p) / p}}{\lambda_{0} p m}\right)^{\alpha /(2-\alpha)} u^{2 /(2-\alpha)}(1+o(1)) .
\end{aligned}
$$

Мы получили соотношение (1.18). Четвертое утверждение теоремы 1 доказано.

\section{7. ДОКАЗАТЕЛЬСТВО ТЕОРЕМ 5, 6 И ПРЕДЛОЖЕНИЙ 1 И 2}

Доказательство теоремы 5 проводится по той же схеме, что и доказательство теоремы 1. При этом, как будет видно из приведенного ниже изложения, ключевые рассуждения и выводы, используемые при доказательстве теоремы 5, очень близки к соответствующим рассуждениям и выводам, полученным при доказательстве теоремы 1. Мы докажем теорему 5, применяя теоремы 7 и 9. Используем масштабное свойство процесса Боголюбова из предложения 3 для интегралов из теоремы 5. Имеет место следующее утверждение.

ЛЕмма 19. Для любых $p>0, u>0$ выполнено равенство

$$
\mathbf{E}\left[\int_{0}^{\beta}|\xi(t)|^{p} d t\right]^{u}=\left(\frac{\beta}{m}\right)^{u p / 2} \beta^{u} \mathbf{E}\left[\int_{0}^{1}|\eta(s)|^{p} d s\right]^{u} .
$$

ДокАзАтЕльство. Сделав в первом интеграле в (7.1) замену переменной $t=\beta s$ и используя формулу (4.1), получаем равенство (7.1).

Таким образом, нам достаточно доказать вариант теоремы 5 для процесса Боголюбова $\eta(t)$ с параметрами $\left(1,1, \omega_{1}\right)$. 
7.1. Выбор банахова функционального пространства. Выбор пространства для доказательства теоремы 5 мы осуществляем точно так же, как в п. 6.1 при доказательстве теоремы 1. Ниже мы используем обозначения и соглашения п. 6.1, в частности формулу (6.2). Таким образом, в данном разделе мы вновь рассматриваем гауссовскую меру Боголюбова $\mu_{\eta}$, заданную в пространстве $L^{\tilde{p}}[0,1]$, имея в виду, что эта мера сосредоточена на $C^{0}[0,1]$. Как уже было сказано, в этом случае, мера $\mu_{\eta}$ имеет нулевое среднее значение и инъективный ковариационный оператор $A_{\eta}: L^{\tilde{q}} \rightarrow L^{\tilde{p}}$ интегрального типа, который определяется по формуле (4.5) для $x \in L^{\tilde{q}}$.

7.2. Выбор основного функционала. Мы докажем теорему 5 , применяя теоремы 7 и 9 , где мы будем полагать $E=L^{\tilde{p}}, P_{A}=\mu_{\eta}, A=A_{\eta}$ и $f \equiv 1$.

Возьмем в качестве функционала $F$ из теорем 7 и 9 следующий функционал:

$$
F(x):=-p \ln \|x\|_{p} \equiv-\ln \left(\int_{0}^{1}|x(t)|^{p} d t\right), \quad p>0, \quad x \in L^{\tilde{p}}
$$

Наша цель - применить теоремы 7 и 9 к данному функционалу и гауссовской мере Боголюбова $\mu_{\eta}$. Используя определение (7.2) и свойство однородности, запишем последнее среднее из формулы (7.1) в виде модельного интеграла Лапласа:

$$
\mathbf{E}\left[\int_{0}^{1}|\eta(t)|^{p} d t\right]^{u}=u^{u p / 2} \mathbf{E} \exp \left\{-u F\left(\frac{\eta}{\sqrt{u}}\right)\right\}
$$

Видно, что роль большого параметра из теорем 7 и 9 теперь играет число $\sqrt{u}$.

ЛЕмма 20. Для функиионала (7.2) и ковариационного оператора $A_{\eta}$, заданного в (4.5) для $x \in L^{\tilde{q}}$, выполнено условие D1.

ДокАзАТЕЛьСтво несложно провести, используя неравенства (1.51) при $\beta=1$.

Функционал $-F(x)$ согласно (7.2) представляет собой логарифм интегрального функционала. Как уже было указано в разделе 6 , интегральные функционалы дифференцируемы по Фреше [42], [56], [57]. Как и ранее, через $\mathcal{X}$ обозначено множество функций из $C^{0}[0,1]$, имеющих конечное число нулей.

ЛЕмма 21. Функиионал (7.2) триждь дифферениируем по Фреше в каждой точке $x \in \mathcal{X}$, при этом первые две производные имеют следующий вид:

$$
\begin{aligned}
F^{\prime}(x)= & -p\|x\|_{p}^{-p}|x(t)|^{p-1} \operatorname{sign} x(t), \\
F^{\prime \prime}(x) g= & p(1-p)\|x\|_{p}^{-p}|x(t)|^{p-2} g+ \\
& \quad+p^{2}\|x\|_{p}^{-2 p}|x(t)|^{p-1} \operatorname{sign} x(t)\left\langle g,|x(t)|^{p-1} \operatorname{sign} x(t)\right\rangle, \quad g \in L^{\tilde{p}} .
\end{aligned}
$$

При $р \geqslant 2$ операторы (7.4) являются ограниченными.

ДокАЗАТЕЛЬство получаем, применяя теорему о суперпозиции дифференцируемых отображений [57] и известные формулы для первых двух производных функционала $\widehat{F}(x)=\int_{0}^{1}|x(t)|^{p} d t$. 
7.3. Решение экстремальной задачи. Решим экстремальную задачу из условия D2 для функционала (7.2) и ковариационного оператора $A_{\eta}$. По аналогии с (6.8)-(6.11) введем следующие величины:

$$
\begin{gathered}
c_{2}=c_{2}(p)=\frac{\sqrt{p}}{\omega_{1}}, \quad e_{2}(t):=c_{2} e_{0}(t) \equiv \frac{\sqrt{p}}{\omega_{1}}, \quad 0<p \leqslant p_{0}, \quad t \in[0,1], \\
c_{3}=c_{3}(p)=\frac{1}{\sqrt{\lambda_{0}}}, \quad p>p_{0},
\end{gathered}
$$

где числа $p_{0}, \lambda_{0}$ заданы в формулах $(1.2),(1.10)$, а функции $e_{0}(t)$ - в формуле $(5.5)$. Положим для $p>0$

$$
\tilde{q}_{2}=\tilde{q}_{2}(p)=\frac{p}{2}-\frac{p}{2} \ln \left(\frac{p}{\omega_{1}^{2}}\right) .
$$

Отметим, что число $\tilde{q}_{2}$ получается из числа $q_{2}$, заданного в $(1.37)$, если в $(1.37)$ положить $m=1, \beta=1, \omega=\omega_{1}$. Этот факт согласуется со связью между процессами $\xi(t)$ и $\eta(t)$, установленной в предложении 3.

ЛЕмма 22. Имеют место следующие утверждения.

1. Пусть фиксировано число $0<p \leqslant p_{0}$. Тогда минимум в экстремальной задаче

$$
\frac{1}{2}\left\langle x, A_{\eta}^{-1} x\right\rangle+F(x) \rightarrow \inf , \quad x \in \operatorname{Dom}\left(A_{\eta}^{-1}\right),
$$

равен $\tilde{q}_{2}$ и достигается на двух функииях $\pm e_{2}(t)$. При этом минимум

$$
\inf _{x \in H_{A_{\eta}}}\left[F(x)+\frac{1}{2}\|x\|_{A_{\eta}}^{2}\right]
$$

также достигается только в двух точках $\pm e_{2}(t)$.

2. Пусть фиксировано число $p>p_{0}$. Тогда минимум в экстремальной задаче (7.8) равен $q_{3}$ и достигается на одномерном компактном четырехсвязном многообразии

$$
\mathcal{V}:=c_{3} \mathcal{M}=\left\{c_{3} x: x \in \mathcal{M}\right\} \subset \operatorname{Ran}\left(A_{\eta}\right),
$$

где $q_{3}$ задано в формуле (1.44), а многообразие $\mathcal{M}$ - в (5.16). Минимум (7.9) также достигается только на множестве $\mathcal{V}$.

ДокАЗАТЕЛьство аналогично доказательству леммы 18. Изложим его. Вывод обоих утверждений леммы основан на лемме 10 и проводится единым образом. Переходя к условной экстремальной задаче, убеждаемся, что справедливы следующие равенства:

$$
\begin{aligned}
U & :=\inf _{x \in \operatorname{Dom}\left(A_{\eta}^{-1}\right)}\left[\frac{1}{2}\left\langle x, A_{\eta}^{-1} x\right\rangle-p \ln \|x\|_{p}\right]= \\
& =\inf _{c>0} \inf _{\substack{x \in \operatorname{Dom}\left(A_{\eta}^{-1}\right) \\
\|x\|_{p}=c}}\left[\frac{1}{2}\left\langle x, A_{\eta}^{-1} x\right\rangle-p \ln \|x\|_{p}\right]=\inf _{c>0} \inf _{\substack{\left.x \in \operatorname{Dom}_{\|x\|_{p}=c}^{-1}\right) \\
\| x}}\left[\frac{1}{2}\left\langle x, A_{\eta}^{-1} x\right\rangle-p \ln c\right] .
\end{aligned}
$$


Внутренняя экстремальная задача в последнем выражении в (7.11) решена в (6.16), поэтому в силу (6.16) при фиксированном $c>0$ имеет место равенство

$$
\inf _{\substack{x \in \operatorname{Dom}\left(A_{\eta}^{-1}\right) \\\|x\|_{p}=c}} \frac{1}{2}\left\langle x, A_{\eta}^{-1} x\right\rangle=\frac{c^{2}}{2 \sigma^{2}}
$$

где:

1) при $0<p \leqslant p_{0}$ минимум в экстремальной задаче в $(7.12)$ достигается на двух функциях $\pm x^{c}(t):= \pm c e_{0}(t)$, а $\sigma^{2}=\sigma_{1}^{2}:=1 / \omega_{1}^{2}$;

$2)$ при $p>p_{0}$ минимум в экстремальной задаче в (7.12) достигается на одномерном многообразии $\mathcal{S}_{c}:=c \mathcal{M}=\{c x: x \in \mathcal{M}\} \subset \operatorname{Ran}\left(A_{\eta}\right)$, а $\sigma^{2}=\sigma_{0}^{2}:=1 / \lambda_{0} p$. Таким образом, из соотношений $(7.11),(7.12)$ получаем равенства

$$
U=\inf _{c>0}\left(\frac{c^{2}}{2 \sigma^{2}}-p \ln c\right)=\frac{c_{\min }^{2}}{2 \sigma^{2}}-p \ln c_{\min }=\frac{p}{2}-\frac{p}{2} \ln \left(p \sigma^{2}\right),
$$

где $c_{\min }=\sigma \sqrt{p}$. Используя формулы $(1.44),(7.5)-(7.7)$ и (7.10)-(7.13), убеждаемся, что при $0<p \leqslant p_{0}$ мы имеем $c_{\min }=c_{2}, U=\tilde{q}_{2}$, а при $p>p_{0}$ выполнены равенства $c_{\text {min }}=c_{3}, U=q_{3}, \mathcal{S}_{c_{3}}=\mathcal{V}$. Отсюда с учетом хороших свойств гильбертова пространства $H_{A_{\eta}}$ и свойства гладкости функционала $F$ вытекают оба утверждения леммы.

7.4. Спектр интегрального оператора $A_{\eta} F^{\prime \prime}\left(e_{2}\right)$ при $0<p \leqslant p_{0}$. При $0<p \leqslant p_{0}$ вследствие лемм 20-22 гауссовская мера Боголюбова $\mu_{\eta}$ и функционал (7.2) удовлетворяют условиям D1-D3 теоремы 7 при $E=L^{\tilde{p}}, f \equiv 1$ и с двумя экстремальными точками $\pm e_{2}$.

Рассмотрим условие D4. Согласно (7.5) мы имеем $F^{\prime \prime}\left(e_{2}\right)=F^{\prime \prime}\left(-e_{2}\right)$, поэтому достаточно исследовать спектр интегрального оператора $A_{\eta} F^{\prime \prime}\left(e_{2}\right): L^{\tilde{p}} \rightarrow L^{\tilde{p}}$. Этот оператор является ядерным как композиция ядерного оператора $A_{\eta}$ и ограниченного оператора $F^{\prime \prime}\left(e_{2}\right)$ [60].

Учитывая формулы (7.4), (7.5), получаем соотношение

$$
\left[F^{\prime \prime}\left(e_{2}\right) z\right](t)=(1-p) \omega_{1}^{2} z(t)+p \omega_{1}^{2} \int_{0}^{1} z(s) d s, \quad t \in[0,1], \quad z \in L^{\tilde{p}}
$$

ЛЕмма 23. При $0<p \leqslant p_{0}$ собственные числа $\left\{\gamma_{n}\right\}_{n=-\infty}^{\infty}$ ядерного интегрального оператора $A_{\eta} F^{\prime \prime}\left(e_{2}\right)$ имеют следующий вид:

$$
\gamma_{0}=1, \quad \gamma_{n}=\frac{(1-p) \omega_{1}^{2}}{\omega_{1}^{2}+(2 \pi n)^{2}}, \quad n= \pm 1, \pm 2, \ldots .
$$

ДокАЗАТЕЛЬСТво проводится аналогично доказательству леммы 5 из [30] при помощи леммы 7 и формул (4.7), (7.14).

Определим оператор $R=I+A_{\eta} F^{\prime \prime}\left(e_{2}\right): L^{\tilde{p}} \rightarrow L^{\tilde{p}}$; этот оператор в рассматриваемой нами задаче играет роль оператора $R_{1}$ из условия $\mathbf{D} 4$. 


\section{5. Вычисление определителя оператора $R$.}

ЛЕмма 24. Справедливы следующие утверждения.

1. Предположим, что фиксировано число $0<p<2$. Тогда имеет место равенство

$$
\operatorname{det} R \equiv \prod_{n=-\infty}^{\infty}\left(1+\gamma_{n}\right)=\frac{2 \operatorname{sh}^{2}\left(\frac{\omega_{1}}{2} \sqrt{2-p}\right)}{(2-p) \operatorname{sh}^{2} \frac{\omega_{1}}{2}}
$$

2. При $p=2$ выполнено равенство

$$
\operatorname{det} R=\frac{\omega_{1}^{2}}{2 \operatorname{sh}^{2} \frac{\omega_{1}}{2}} .
$$

3. Пусть фиксировано число $0<p \leqslant p_{0}$. Тогда

$$
\operatorname{det} R=\frac{2 \sin ^{2}\left(\frac{\omega_{1}}{2} \sqrt{p-2}\right)}{(p-2) \operatorname{sh}^{2} \frac{\omega_{1}}{2}} .
$$

4. Для оператора $A_{\eta} F^{\prime \prime}\left(e_{2}\right)$ выполнено условие D4 при всех $0<p<p_{0}$.

ДокАЗАТЕЛьство первых трех утверждений проводится аналогично доказательству леммы 15 из [30]. Четвертое утверждение вытекает из первых трех и того факта, что детерминант (7.15) равен нулю при $p=p_{0}$.

7.6. Доказательство п. 1-3 теоремы 5. Найдем точную асимптотику при $u \rightarrow \infty$ для последнего интеграла Лапласа в (7.3). Пусть фиксировано число $0<p<p_{0}$. Тогда вследствие лемм 20-24 гауссовская мера Боголюбова $\mu_{\eta}$ и функционал (7.2) удовлетворяют условиям D1-D4 теоремы 7 при $E=L^{\tilde{p}}, f \equiv 1, m=2$, $x^{1}=e_{2}$ и $x^{2}=-e_{2}$. Таким образом, к последнему интегралу из формулы (7.3) применима теорема 7, и мы можем использовать соотношение (2.1) с большим параметром $\sqrt{u}$ вместо $u$. Несложно видеть, что константы из правой части формулы (2.1) для обеих экстремальных функций совпадают, поэтому достаточно провести вычисления только для точки $e_{2}$. Используя леммы 22,24 и формулы $(2.1),(7.3),(7.7)$, мы получаем утверждения 1-3 теоремы 5 для гауссовского процесса Боголюбова $\eta(t)$. Затем, учитывая уже доказанные формулы и равенства (4.2), (7.1), мы получаем соотношения (1.38)-(1.42) теоремы 5. Тем самым три первых утверждения теоремы 5 доказаны.

7.7. Доказательство п. 4 теоремы 5. Докажем четвертое утверждение теоремы 5 при $p>p_{0}$, используя п. 3 теоремы 9 , где $E=L^{p}[0,1], P_{A}=\mu_{\eta}, A=A_{\eta}$, а функционал $F$ задан в (7.2). Как указано в лемме 20 , функционал $F$ удовлетворяет условию D1, поэтому для него выполнено соотношение (2.5). Применяя соотношение (2.5) с учетом замечания 7, формул (1.44), (7.1), (7.3) и п. 2 леммы 22, получаем следующую цепочку равенств при $p>p_{0}$ и $u \rightarrow \infty$ :

$$
\begin{aligned}
\mathbf{E}\left[\int_{0}^{\beta}|\xi(t)|^{p} d t\right]^{u} & =\left(\frac{\beta u}{m}\right)^{u p / 2} \beta^{u} \mathbf{E} \exp \left\{-u F\left(\frac{\eta}{\sqrt{u}}\right)\right\}= \\
& =\left(\frac{\beta u}{m}\right)^{u p / 2} \beta^{u} \exp \left\{-u \inf _{x \in L^{p}}\left[F(x)+I_{A_{\eta}}(x)\right](1+o(1))\right\}= \\
& =\left(\frac{\beta u}{m}\right)^{u p / 2} \beta^{u} e^{-u q_{3}(p)(1+o(1))}
\end{aligned}
$$

Мы получили соотношение (1.43). Четвертое утверждение теоремы 5 доказано. 
7.8. Доказательство теоремы 6. Доказательство теоремы 6 проводится по той же схеме, что и доказательство первых трех утверждений теоремы 5 на основе теоремы 7. При этом существенным образом используются утверждения, уже полученные в настоящем разделе. Укажем основные моменты. Пусть выполнены условия теоремы 6 . В качестве банахова пространства $E$, участвующего в формулировке теоремы 7 , возьмем вновь пространство $L^{\tilde{p}}$, где число $\tilde{p}$ задано в формуле (6.2). В качестве функционала $F$ возьмем функционал

$$
F_{1}(x):=-\ln \left|\int_{0}^{1} x^{p}(t) d t\right|, \quad 0<p<p_{0}, \quad x \in L^{\tilde{p}} .
$$

Тогда в силу предложения 3 справедлив следующий аналог первого равенства в (7.16) при $u=2 k$ :

$$
\mathbf{E}\left[\int_{0}^{\beta} \xi^{p}(t) d t\right]^{2 k}=\mathbf{E}\left|\int_{0}^{\beta} \xi^{p}(t) d t\right|^{2 k}=\left(\frac{2 k \beta}{m}\right)^{k p} \beta^{2 k} \mathbf{E} \exp \left\{-2 k F_{1}\left(\frac{\eta}{\sqrt{2 k}}\right)\right\} .
$$

Функционал $F_{1}$ трижды дифференцируем по Фреше в каждой точке $x \in \mathcal{X}_{0}$, где

$$
\mathcal{X}_{0}:=\left\{x \in \mathcal{X}: \int_{0}^{1} x^{p}(t) d t \neq 0\right\} .
$$

В случае функционала (7.17) минимум в экстремальной задаче из условия D2, т. е. в задаче

$$
\frac{1}{2}\left\langle x, A_{\eta}^{-1} x\right\rangle+F_{1}(x) \rightarrow \inf , \quad x \in \operatorname{Dom}\left(A_{\eta}^{-1}\right),
$$

равен $\tilde{q}_{2}$ и достигается на двух функциях $\pm e_{2}(t)$. В этом можно убедиться, просматривая доказательство леммы 22 и учитывая, что $F_{1}\left(e_{2}\right)=F_{1}\left(-e_{2}\right)=F\left(e_{2}\right)$, $F_{1}^{\prime}\left(e_{2}\right)=-F_{1}^{\prime}\left(-e_{2}\right)=F^{\prime}\left(e_{2}\right)$, где функционал $F$ задан в $(7.2)$. Кроме того, для функционалов (7.2) и (7.18) справедливы равенства $F_{1}^{\prime \prime}\left(e_{2}\right)=F_{1}^{\prime \prime}\left(-e_{2}\right)=F^{\prime \prime}\left(e_{2}\right)$, поэтому утверждения лемм 23,24 остаются справедливыми и для оператора $R_{1}:=$ $I+A_{\eta} F_{1}^{\prime \prime}\left(e_{2}\right) \equiv R$. Таким образом, в условиях теоремы 6 применима теорема 7 с $E=L^{\tilde{p}}, f \equiv 1, F=F_{1}, m=2, x^{1}=e_{2}, x^{2}=-e_{2}$ и большим параметром $u=\sqrt{2 k} \rightarrow \infty$. Далее в условиях теоремы 6 следует рассмотреть отдельно три случая значений параметра $p$, а именно $0<p<2, p=2,2<p<p_{0}$. Применяя во всех трех случаях теорему 7 и учитывая теорему 5 , приходим к формуле (1.45). Теорема 6 доказана.

7.9. Доказательство предложений 1 и 2. Доказательство соотношений (1.33), (1.35) и (1.49) проводится при помощи формул (2.4), (2.5) из теоремы 9 и первых пунктов лемм 10, 18, 22 аналогично доказательству четвертых утверждений теорем 1, 2 и 5. Доказательство соотношений (1.34), (1.36) и (1.50) проводится также при помощи формул $(2.4),(2.5)$ из теоремы 9 с учетом первых утверждений лемм 10 , 18, 22 аналогично доказательству теоремы 6 с использованием функционалов

$$
G_{1}(x):=\int_{0}^{1} x^{p}(t) d t-1, \quad \Psi_{1}(x):=-\left(\int_{0}^{1} x^{p}(t) d t\right)^{\alpha / p}
$$

и $F_{1}(x)$ из $(7.17)$ (cp. с (5.1) и (6.3)). Некоторые подробности для случаев функционалов $G_{1}(x)$ и $\Psi_{1}(x)$ изложены в статье [30]. 


\section{8. ДОКАЗАТЕЛЬСТВО ТЕОРЕМЫ 3}

Доказательство теоремы 3 основано на следующем асимптотическом результате для гауссовских процессов типа винеровского [15].

Теорема 10. Пусть $\zeta(t), t \in[0, T],-$ гауссовский стационарный процесс с $n . н$. непрерывными траекториями, нулевым средним значением и единичной дисперсией. Предположим, что его ковариачионная функиия $R_{\zeta}(t-s)=\mathbf{E} \zeta(t) \zeta(s), t, s \in$ $[0, T]$, удовлетворяет при некотором с $>0$ условиям

$$
R_{\zeta}(t)=1-c|t|+o(|t|), \quad t \rightarrow 0, \quad-1<R_{\zeta}(t)<1, \quad t \in(0, T] .
$$

Тогда при и $\rightarrow \infty$ выполнены соотношения

$$
\mathbf{P}\left\{\sup _{t \in[0, T]}|\zeta(t)|>u\right\}=2 \mathbf{P}\left\{\sup _{t \in[0, T]} \zeta(t)>u\right\}(1+o(1))=e^{-u^{2} / 2} u \frac{c T \sqrt{2}}{\sqrt{\pi}}(1+o(1)) .
$$

Доказательство данной теоремы можно получить, используя следствие 6.4 и теорему 7.1 из монографии [15] при $n=1, \alpha=1$, учитывая что $H_{1}=1$.

8.1. Доказательство соотношения (1.28) из теоремы 3. Пусть фиксировано число $0<\beta_{0}<\beta$. Используя масштабное свойство процесса Боголюбова из предложения 3 , получаем следующее равенство для $u>0$ :

$$
\mathbf{P}\left\{\sup _{t \in\left[0, \beta_{0}\right]} \xi(t)>u\right\}=\mathbf{P}\left\{\sup _{t \in\left[0, \beta_{0} / \beta\right]} \eta(t)>v\right\}, \quad v=\frac{\sqrt{m}}{\sqrt{\beta}} u .
$$

Учитывая равенства (4.2), (4.3), получаем формулу для дисперсии стационарного гауссовского процесса $\eta(t)$,

$$
\sigma_{\eta}^{2}:=\mathbf{E} \eta^{2}(t)=\frac{\operatorname{ch} \frac{\omega_{1}}{2}}{2 \omega_{1} \operatorname{sh} \frac{\omega_{1}}{2}}, \quad t \in[0,1],
$$

и его корреляционной функции,

$$
r_{\eta}(t-s):=\frac{\mathbf{E} \eta(t) \eta(s)}{\sigma_{\eta}^{2}}=\frac{\operatorname{ch}\left(\omega_{1}|t-s|-\frac{\omega_{1}}{2}\right)}{\operatorname{ch} \frac{\omega_{1}}{2}}, \quad t, s \in[0,1] .
$$

Используя последнее соотношение, несложно получить разложение

$$
r_{\eta}(t)=1-\frac{|t|}{2 \sigma_{\eta}^{2}}+o(|t|), \quad t \rightarrow 0
$$

и оценку $0<r_{\eta}(t)<1, t \in\left(0, \beta_{0} / \beta\right]$. Отсюда мы заключаем, что к процессу $\eta(t) / \sigma_{\eta}$, $t \in\left[0, \beta_{0} / \beta\right]$, применима теорема 10 при $T=\beta_{0} / \beta, c=1 / 2 \sigma_{\eta}^{2}$. Используя формулы (8.2) и (8.3), получаем следующие соотношения при $u \rightarrow \infty$ :

$$
\mathbf{P}\left\{\sup _{t \in\left[0, \beta_{0}\right]} \xi(t)>u\right\}=\mathbf{P}\left\{\sup _{t \in\left[0, \beta_{0} / \beta\right]} \frac{\eta(t)}{\sigma_{\eta}}>\frac{v}{\sigma_{\eta}}\right\}=e^{-v^{2} / 2 \sigma_{\eta}^{2}} v \frac{\beta_{0}}{2 \beta \sigma_{\eta}^{3} \sqrt{2 \pi}}(1+o(1)) .
$$


Учитывая формулы $(8.4),(8.7)$ и равенство $v=(\sqrt{m} / \sqrt{\beta}) u$, убеждаемся, что при $u \rightarrow \infty$ справедливо второе соотношение в формуле (1.28). Первое соотношение в (1.28) вытекает из формулы

$$
\mathbf{P}\left\{\sup _{t \in\left[0, \beta_{0}\right]}|\xi(t)|>u\right\}=\mathbf{P}\left\{\sup _{t \in\left[0, \beta_{0} / \beta\right]}|\eta(t)|>v\right\}=\mathbf{P}\left\{\sup _{t \in\left[0, \beta_{0} / \beta\right]} \eta(t)>v\right\}(1+o(1))
$$

и соотношения (8.7). Утверждение (1.28) доказано.

Брать в формуле (1.28) в качестве $\beta_{0}$ число $\beta$, вообще говоря, нельзя. Это объясняется тем, что $r_{\eta}(1)=1$ согласно формуле (8.5), и теорема 10 неприменима к процессу $\eta(t) / \sigma_{\eta}$ при $t \in[0,1]$, так как в этом случае нарушается условие $(8.1)$ в крайней точке $t=1$.

8.2. Доказательство соотношения (1.29) из теоремы 3. Используя масштабное свойство процесса Боголюбова из предложения 3, получаем следующее равенство для $u>0$ :

$$
\mathbf{P}\left\{\sup _{t \in[0, \beta]} \xi(t)>u\right\}=\mathbf{P}\left\{\sup _{t \in[0,1]} \eta(t)>v\right\}, \quad v=\frac{\sqrt{m}}{\sqrt{\beta}} u .
$$

Применяя известный результат Ландау-Маркуса-Шеппа (см., например, [61]), получаем соотношение

$$
\lim _{v \rightarrow \infty} \frac{1}{v^{2}} \ln \mathbf{P}\left\{\sup _{t \in[0,1]} \eta(t)>v\right\}=-\frac{1}{2 \mathbf{E} \eta^{2}(t)} .
$$

Учитывая формулы (8.4), (8.8), (8.9), убеждаемся, что справедливо равенство

$$
\lim _{u \rightarrow \infty} \frac{1}{u^{2}} \ln \mathbf{P}\left\{\sup _{t \in[0, \beta]} \xi(t)>u\right\}=-m \omega \operatorname{th}\left(\frac{\beta \omega}{2}\right) .
$$

Аналогично доказывается второе равенство в (1.29). Теорема 3 доказана.

\section{Список литературы}

[1] Р. Фейнман, "Пространственно-временной поход к нерелятивистской квантовой механике", Вопросы причинности в квантовой механике, ред. Я. П. Терлецкий, А. А. Гусев, ИЛ, М., 1955, 167-207.

[2] Р. Фейнман, А. Хибс, Квантовая механика и интегралы по траекториям, Мир, М., 1968.

[3] S. Albeverio et al. (eds.), Feynman Path integrals, Proc. International Colloquium (Marseille, May 1978), Lect. Notes Phys., 106, Springer, Berlin, Heidelberg, New York, 1979.

[4] F. W. Wiegel, Phys. Rep., 16:2 (1975), 57-114.

[5] В. Н. Попов, Континуальные интеграль в квантовой теории поля и статистической физике, Атомиздат, М., 1976.

[6] B. Simon, Functional Integration and Quantum Physics, Lect. Notes Pure Appl. Math., 86, Academic Press, New York, London, 1979.

[7] L.S. Schulman, Techniques and applications of path integration, Wiley, New York, 1981.

[8] Дж. Глимм, А. Джаффе, Математические методы квантовой физики. Подход с использованием континуальных интегралов, Мир, М., 1984. 
[9] G. W. Johnson, M. L. Lapidus, The Feynman Integral and Feynman's Operational Calculus, Clarendon Press, Oxford, 2000.

[10] П. Л. Рубин, ТМФ, 156:2 (2008), 184-188.

[11] Л.А. Янович, Приближенное вычисление континуальных интегралов по гауссовым мерам, Наука и техника, Минск, 1976.

[12] И. М. Ковальчик, Л. А. Янович, Обобщенный винеровский интеграл и некоторые его приложения, Наука и техника, Минск, 1989.

[13] R. S. Ellis, J. S. Rosen, Comm. Math. Phys, 82:2 (1981), 153-181.

[14] R. S. Ellis, J. S. Rosen, Trans. Amer. Math. Soc., 273:2 (1982), 447-481.

[15] В. И. Питербарг, Асимптотические методы в теории гауссовских случайных процессов и полей, Изд-во МГУ, М., 1988.

[16] В. И. Питербарг, В. Р. Фаталов, УМН, 50:6 (1995), 57-150.

[17] В. Р. Фаталов, “Точные асимптотики винеровских интегралов типа Лапласа для $L^{p}$-функционалов", Изв. РАН. Сер. матем., 74:1 (2010), 197-224.

[18] Н. Н. Боголюбов, Докл. АН СССР, 99:2 (1954), 225-226; Собр. научных трудов, т. 9: Квантовая теория поля, ред. А. Д. Суханов, Наука, М., 2007.

[19] Н. Н. Боголюбов, Н. Н. Боголюбов (мл.), Аспекты теории полярона, Физматлит, М., 2004.

[20] Д. П. Санкович, ТМФ, 119:2 (1999), 345-352.

[21] Д. П. Санкович, ТМФ, 126:1 (2001), 149-163.

[22] Д. П. Санкович, ТМФ, 127:1 (2001), 125-142.

[23] Д. П. Санкович, “Функциональный интеграл Боголюбова”, Нелинейная динамика, Сборник статей, Тр. МИАН, 251, Наука, М., 2005, 223-256.

[24] Г. Крамер, М. Лидбеттер, Стационарные случайнъе процессы, Мир, М., 1969.

[25] И. И. Гихман, А. В. Скороход, Теория случайных процессов, т. 1, Наука, М., 1971.

[26] М. А. Лифшиц, Гауссовские случайные функиии, ТВиМС, Киев, 1995.

[27] Х.-С. Го, Гауссовские меры в банаховых пространствах, Мир, М., 1979.

[28] В. И. Богачев, Гауссовские меры, Физматлит, М., 1997.

[29] Н.Н.Вахания, В.И. Тариеладзе, С. А. Чобанян, Вероятностные распределения в банаховых пространствах, Наука, М., 1985.

[30] В. Р. Фаталов, ТМФ, 157:2 (2008), 286-308.

[31] E. Brézin, J. C. Le Guillou, J. Zinn-Justin, Phys. Rev. D, 15:6 (1997), 1544-1557.

[32] В. Р. Фаталов, Проблемы перед. информаи., 42:1 (2006), 52-71.

[33] В. Р. Фаталов, Проблемы перед. информаи., 46:1 (2010), 68-93.

[34] М.В. Федорюк, Асимптотика: интеграль и ряды, Наука, М., 1987.

[35] N. Bleistein, R. A. Handelsman, Asymptotic Expansions of Integrals, Holt, Rinehart and Winston, New York, 1975.

[36] K.W. Breitung, Asymptotic Approximations for Probability Integrals, Lect. Notes Math., 1592, Springer, Berlin, 1994.

[37] В. Р. Фаталов, "Метод Лапласа для гауссовских мер в банаховом пространстве (многообразие точек минимума) с применением к статистике Ватсона", ТВП, в печати.

[38] М. Кац, Вероятность и смежные вопросы в физике, Наука, М., 1965.

[39] А.Н. Ширяев, Вероятность - 1, Изд-во МЦНМО, М., 2004.

[40] М. Абрамовиц, И. Стиган (ред.), Справочник по специальным функциям, Наука, М., 1979.

[41] Л.В.Канторович, Г. П. Акилов, Функииональный анализ, Наука, М., 1977.

[42] М.А. Красносельский, П. П. Забрейко, Е. И. Пустыльник, П. Е. Соболевский, Интегральные операторы в пространствах суммируемых функиий, Наука, М., 1966. 
[43] В. Р. Фаталов, "Моменты $L^{p}$-функционалов от гауссовских процессов: точные асимптотики", в печати.

[44] V. R. Fatalov, J. Contemp. Math. Anal., 27:5 (1992), 36-50.

[45] S. R. S. Varadhan, Commun. Pure Appl. Math., 19:3 (1966), 261-286.

[46] S. R. S. Varadhan, Ann. Probab., 36:2 (2008), 397-419.

[47] J.-D. Deuschel, D. W. Stroock, Large Deviations, Pure Appl. Math., 137, Acad. Press, Boston, MA, 1989.

[48] A. Dembo, O. Zeitouni, Large Deviations Techniques and Applications, Appl. Math., 38, Springer, New York, 1998.

[49] J. A. Bucklew, Large Deviation Techniques in Decision, Simulation and Estimation, Wiley, New York, 1990.

[50] Х.-О. Георги, Гиббсовские меры и фазовые переходъ, Мир, М., 1992.

[51] C.-E. Pfister, "Thermodynamical aspects of classical lattice systems", In and Out of Equilibrium, 4th Brazilian school of probability (Mambucaba, Brazil, August 14-19, 2000), Progr. Probab., 51, ed. V. Sidoravicius, Birkhäuser, Boston, 2002, 393-472.

[52] E. Olivieri, M. E. Vares, Large Deviations and Metastability, Encyclopedia of Mathematics and its Applications, 100, Cambridge Univ. Press, Cambridge, 2005.

[53] R. S. Ellis, Entropy, Large Deviations, and Statistical Mechanics, Springer, Berlin, 2006.

[54] Э. Камке, Справочник по обыкновенным дифференциалъным уравнениям, Наука, М., 1961.

[55] Э.А. Коддингтон, Н. Левинсон, Теория обыкновенных дифференииальных уравнений, УPCC, M., 2007.

[56] М. М. Вайнберг, Вариационный метод и метод монотонных операторов в теории нелинейных уравнений, Наука, М., 1972.

[57] В. М. Алексеев, В. М. Тихомиров, С. В. Фомин, Оптимальное управление, Наука, М., 1979.

[58] С. Г. Крейн (ред.), Функиионалъныц анализ, Наука, М., 1972.

[59] Ф. Рисс, Б. Сёкефальви-Надь, Лекиии по функииональному анализу, Мир, М., 1979.

[60] А. Пич, Операторные идеальь, Мир, М., 1982.

[61] R. J. Adler, An Introduction to Continuity, Extrema, and Related Topics for General Gaussian Processes, IMS Lect. Notes, 12, Hayward, CA, IMS, 1990. 